\title{
THE LEBEAU-ROBBIANO INEQUALITY FOR THE ONE-DIMENSIONAL FOURTH ORDER ELLIPTIC OPERATOR AND ITS APPLICATION
}

\author{
PENG GaO ${ }^{1,2}$
}

\begin{abstract}
In this paper, we establish the Lebeau-Robbiano inequality for the one-dimensional fourth order elliptic operator by using a point-wise estimate. Based on this inequality, we obtain the null controllability of one-dimensional stochastic fractional order Cahn-Hilliard equation.
\end{abstract}

Mathematics Subject Classification. 93B05, 35K35, $60 \mathrm{H} 15$.

Received August 14, 2014. Revised April 25, 2015.

Published online May 27, 2016.

\section{INTRODUCTION}

In this paper, we investigate the one-dimensional fourth order elliptic operator $A$ on $L^{2}(I)$ as follows

$$
\left\{\begin{array}{l}
\mathcal{D}(A)=H_{0}^{2}(I) \cap H^{4}(I), \\
A y=y_{x x x x} \quad \forall y \in \mathcal{D}(A),
\end{array}\right.
$$

where $I=(0,1)$.

Let $\left\{\lambda_{i}\right\}_{i=1}^{\infty}, 0<\lambda_{1} \leq \lambda_{2} \leq \ldots$ be the eigenvalues of $A$ and $\left\{e_{i}\right\}_{i=1}^{\infty}$ be the corresponding real eigenfunctions such that $\left\|e_{i}\right\|_{L^{2}(I)}=1(i=1,2,3, \ldots)$, which serves as an orthonormal basis of $L^{2}(I)$ (see [13], Thm. 8.94).

The main result in this paper is an observability estimate on partial sums of eigenfunctions for the eigenfunctions of $A$, i.e. the Lebeau-Robbiano inequality:

Theorem 1.1. Let $\omega$ be a nonempty open subset of $I$. There exist two positive constants $C_{1}, C_{2}$ such that

$$
\sum_{\lambda_{i} \leq r}\left|a_{i}\right|^{2} \leq C_{1} \mathrm{e}^{C_{2} \sqrt{r}} \int_{\omega}\left|\sum_{\lambda_{i} \leq r} a_{i} e_{i}\right|^{2} \mathrm{~d} x
$$

for every finite $r>0$ and every choice of $\left\{a_{i}\right\}_{\lambda_{i} \leq r}$ with $a_{i} \in \mathbb{C}$.

\footnotetext{
Keywords and phrases. Lebeau-Robbiano inequality, null controllability, stochastic fractional order Cahn-Hilliard equation.

1 School of Mathematics and Statistics, and Center for Mathematics and Interdisciplinary Sciences, Northeast Normal University, Changchun 130024, P.R. China

2 Institute of Mathematics, Jilin University, Changchun 130012, P.R. China. gaopengjilindaxue@126.com
} 
The Lebeau-Robbiano inequality was first established in [5] for the Laplacian with homogeneous Dirichlet boundary condition, then the same result for more general boundary conditions and second order elliptic operators were established in $[7,11]$.

In the study on the controllability of PDE, the inequality of this type is the foundation of Lebeau-Robbiano iteration technology. By this inequality, [5] obtained the null controllability of a linear system of thermoelasticity in a compact, $C^{\infty}, n$-dimensional connected Riemannian manifold. Then, this inequality was used to establish a certain $L^{\infty}$-null controllability for the internal controlled heat equation when the control functions are restricted in arbitrary subset of positive measure in time variable in [14]. Later, in [10], via the Lebeau-Robbiano inequality, the authors presented a time optimal control problem with control constraints of the rectangular type for internal controlled heat equations. Recently, the similar result as in [14] was extended to forward stochastic heat equations in [9] and second order parabolic equation with equivalued surface boundary conditions in [11]. In [3], the Lebeau-Robbiano inequality can also be used to depict nodal sets of sums of functions.

However, to the best of our knowledge, the Lebeau-Robbiano inequality for fourth order elliptic operator has not been established. It should be noted that Theorem 1.1 in this paper is not a straightforward generalization of the Lebeau-Robbiano inequality in [4], although the inequality has the same formal. To prove Theorem 1.1, a point-wise estimate for the operator $\partial_{t}^{2}-\partial_{x}^{4}$ in Proposition 2.1 plays a crucial role. In order to obtain the point-wise estimate, we need to choose suitable grouping for the terms of $\theta\left(y_{t t}-y_{x x x x}\right)$ (see $I_{1}$ and $I_{2}$ in the proof of Prop. 2.1) since the high order of the operator. Due to the special properties of the operator $\partial_{t}^{2}-\partial_{x}^{4}$ and the boundary terms in the point-wise estimate, we need to construct several weight functions.

Based on Theorem 1.1, we can obtain the second result in this paper: the null controllability of one-dimensional stochastic fractional order Cahn-Hilliard equation.

First, we give some assumptions.

(H1) Let $\left(\Omega, \mathcal{F},\left\{\mathcal{F}_{t}\right\}_{t \geq 0}, P\right)$ be a complete filtered probability space on which a one-dimensional standard Brownian motion $\{B(t)\}_{t \geq 0}$ is defined such that $\left\{\mathcal{F}_{t}\right\}_{t \geq 0}$ is the natural filtration generated by $B(\cdot)$, augmented by all the $P$-null sets in $\mathcal{F}$. Let $H$ be a Banach space, and let $C([0, T] ; H)$ be the Banach space of all $H$-valued strongly continuous functionals defined on $[0, T]$. We denote by $L_{\mathcal{F}}^{2}(0, T ; H)$ the Banach space consisting of all $H$-valued $\left\{\mathcal{F}_{t}\right\}_{t \geq 0}$-adapted processes $X(\cdot)$ such that $\mathbb{E}\left(\|X(\cdot)\|_{L^{2}(0, T ; H)}^{2}\right)<\infty$, with the canonical norm; by $L_{\mathcal{F}}^{\infty}(0, T ; H)$ the Banach space consisting of all $H$-valued $\left\{F_{t}\right\}_{t \geq 0}$-adapted bounded processes; and by $\left.L_{\mathcal{F}}^{2}(\Omega ; C[0, T] ; H)\right)$ the Banach space consisting of all $H$-valued $\left\{\mathcal{F}_{t}\right\}_{t \geq 0^{-}}$ adapted continuous processes $X(\cdot)$ such that $\mathbb{E}\left(\|X(\cdot)\|_{C([0, T] ; H)}^{2}\right)<\infty$, with the canonical norm; by $L_{\mathcal{F}}^{r}\left(0, T ; L^{2}(\Omega ; H)\right)$ the Banach space consisting of all $H$-valued $\left\{\mathcal{F}_{t}\right\}_{t \geq 0}$-adapted processes $X$ such that $\|\mathbb{E}\| X\left\|_{H}^{2}\right\|_{L^{r}(0, T)}<\infty(1 \leq r \leq \infty)$, with the canonical norm.

(H2) $E \subset[0, T]$ with positive measure. $\chi_{\omega}$ and $\chi_{E}$ denote the characteristic function of $\omega$ and $E$, respectly.

(H3) We define the operator $A^{\alpha}$ as follows

$$
\left\{\begin{array}{l}
\mathcal{D}\left(A^{\alpha}\right)=\left\{u \in L^{2}(I) \mid u=\sum_{i \geq 1} a_{i} e_{i} \text { and } \sum_{i \geq 1}\left|a_{i}\right|^{2} \lambda_{i}^{2 \alpha}<+\infty\right\}, \\
A^{\alpha} u=\sum_{i \geq 1} a_{i} \lambda_{i}^{\alpha} e_{i} \text { where } u=\sum_{i \geq 1} a_{i} e_{i} .
\end{array}\right.
$$

(H4) $a(t) \in L_{\mathcal{F}}^{\infty}(0, T ; \mathbb{R}), \xi=\|a\|_{L_{\mathcal{F}}^{\infty}(0, T ; \mathbb{R})}^{2}$.

(H5) For each $r>0$, we set $X_{r}=\operatorname{span}\left\{e_{i}(x)\right\}_{\lambda_{i} \leq r}$ and denote by $P_{r}$ the orthogonal projection from $L^{2}(I)$ to $X_{r}$.

(H6) Throughout this paper, $C$ (and sometimes $\widetilde{C}$ ) denotes various positive constants. $C(\ldots)$ stands for a positive constant that depends on what are enclosed in the brackets.

Our second main result in this paper is the following theorem. 
Theorem 1.2. Let $\alpha>\frac{1}{2}$. For any $y_{0} \in L^{2}\left(\Omega, \mathcal{F}_{0}, P ; L^{2}(I)\right)$, there is a control $f \in L_{\mathcal{F}}^{\infty}\left(0, T ; L^{2}\left(\Omega ; L^{2}(I)\right)\right)$ such that the solution $y$ of system

$$
\begin{cases}\mathrm{d} y+A^{\alpha} y=a(t) y \mathrm{~d} B+\chi_{\omega} \chi_{E} f \mathrm{~d} t & \text { in } I \times(0, T), \\ y(0, t)=0=y(1, t) & \text { in }(0, T), \\ y_{x}(0, t)=0=y_{x}(1, t) & \text { in }(0, T), \\ y(x, 0)=y_{0}(x) & \text { in } I,\end{cases}
$$

satisfies $y(T)=0$ in I, P-a.s. Moreover, the control $f$ satisfies the following estimate

$$
\|f\|_{L_{\mathcal{F}}^{\infty}\left(0, T ; L^{2}\left(\Omega ; L^{2}(I)\right)\right)}^{2} \leq L \mathbb{E}\left\|y_{0}\right\|_{L^{2}(I)}^{2},
$$

where $L$ is a constant independent of $y_{0}$.

Remark 1.3. We refer to ([1], Chap. 6) for the well-posedness of system (1.1) in the class $L_{\mathcal{F}}^{2}\left(\Omega ; C\left([0, T] ; L^{2}(I)\right)\right)$.

The rest of this paper is organized as follows. In Section 2, we establish a point-wise estimate. Theorem 1.1 is proved in Section 3 according to two important lemmas. Section 4 is devoted to the proof of Theorem 1.2.

\section{A point-Wise estimate}

In this section, we give a point-wise estimate which will be used in the proof of Theorem 1.1 and the estimate itself has independent interest.

Proposition 2.1. Set $\theta=\mathrm{e}^{l}, l=\lambda \varphi, \varphi=\mathrm{e}^{\mu \psi}$ and $u=\theta y$ in $Q=I \times(0, T)$, where $\psi \in C^{\infty}(Q)$ and $y \in C^{4}(Q)$. Assume that $\mu \geq 1$ and $\lambda \geq \lambda_{0}$, where $\lambda_{0}=\lambda_{0}(\mu, \psi)$ such that $\lambda \mu^{-1} \varphi \geq 1$. Then we have the following point-wise estimate

$$
\begin{aligned}
& \lambda^{7} \mu^{8} \varphi^{7} \psi_{x}^{8} u^{2}+\lambda^{5} \mu^{6} \varphi^{5} \psi_{x}^{6} u_{x}^{2}+\lambda^{3} \mu^{4} \varphi^{3} \psi_{x}^{4} u_{x x}^{2}+\lambda \mu^{2} \varphi \psi_{x}^{2} u_{x x x}^{2} \\
& \quad+\lambda^{3} \mu^{4} \varphi^{3} \psi_{x}^{4} u_{t}^{2}+\lambda \mu^{2} \varphi \psi_{x}^{2} u_{x t}^{2}+\{\ldots\}_{x}+\{\ldots\}_{x x}+\{\ldots\}_{x x x}+\{\ldots\}_{x x x x}+\{\ldots\}_{t}+\{\ldots\}_{t t} \\
& \leq C(\psi)\left(\lambda^{7} \mu^{7} \varphi^{7} u^{2}+\lambda^{5} \mu^{5} \varphi^{5} u_{x}^{2}+\lambda^{3} \mu^{3} \varphi^{3} u_{x x}^{2}+\lambda \mu \varphi u_{x x x}^{2}+\lambda^{3} \mu^{3} \varphi^{3} u_{t}^{2}+\lambda \mu \varphi u_{x t}^{2}+\theta^{2}\left|y_{t t}-y_{x x x x}\right|^{2}\right),
\end{aligned}
$$

where

$$
\begin{aligned}
\{\ldots\}_{x}= & \left\{-\frac{3}{2} B_{2 x x} u_{x}^{2}+\frac{3}{2} B_{2} u_{x x}^{2}-\frac{1}{2} B_{4} u_{x x x}^{2}-4 a_{x} u_{x}^{2}+2 a_{x x x} u^{2}+b_{x} u_{x x}^{2}-B_{0} u_{t} u_{x x x}+\frac{1}{2} B_{1} B_{2} u^{2}\right. \\
& +\frac{3}{2}\left(B_{1} B_{4}\right)_{x x} u^{2}-\frac{3}{2} B_{1} B_{4} u_{x}^{2}-\left(b B_{1}\right)_{x} u^{2}+\frac{1}{2} B_{2} B_{3} u_{x}^{2}+\frac{1}{2} B_{3} B_{4} u_{x x}^{2}-\left(a B_{3}\right)_{x} u^{2}+B_{0} B_{3} u_{t} u_{x} \\
& \left.-\frac{1}{2} B_{2} u_{t}^{2}-\frac{3}{2} B_{4 x x} u_{t}^{2}+\frac{3}{2} B_{4} u_{x t}^{2}-b_{t} u_{t} u_{x}+b_{x} u_{t}^{2}-\left(B_{4} c\right)_{x} u_{x}^{2}+\frac{1}{2} a c u^{2}+\frac{1}{2} b c u_{x}^{2}\right\}_{x}, \\
\{\ldots\}_{x x}= & \left\{\frac{3}{2} B_{2 x} u_{x}^{2}-3 a_{x x} u^{2}+2 a u_{x}^{2}-\frac{b}{2} u_{x x}^{2}-\frac{3}{2}\left(B_{1} B_{4}\right)_{x} u^{2}+\frac{1}{2} b B_{1} u^{2}+\frac{1}{2} a B_{3} u^{2}+\frac{3}{2} B_{4 x} u_{t}^{2}-\frac{1}{2} b u_{t}^{2}+\frac{1}{2} B_{4} c u_{x}^{2}\right\}_{x x}, \\
\{\ldots\}_{x x x}= & \left\{-\frac{1}{2} B_{2} u_{x}^{2}+2 a_{x} u^{2}+\frac{1}{2} B_{1} B_{4} u^{2}-\frac{1}{2} B_{4} u_{t}^{2}\right\}_{x x x}, \\
\{\ldots\}_{x x x x}= & \left\{-\frac{1}{2} a u^{2}\right\}_{x x x x}, \\
\{\ldots\}_{t}= & \left\{\frac{1}{2} B_{0} B_{1} u^{2}-\frac{1}{2} B_{0} B_{3} u_{x}^{2}+B_{2} u_{t} u_{x}+\frac{1}{2} B_{0} u_{t}^{2}-a_{t} u^{2}+B_{4} u_{t} u_{x x x}+b u_{t} u_{x x}+\frac{1}{2} b_{t} u_{x}^{2}\right\}_{t}, \\
\{\ldots\}_{t t}= & \left\{\frac{1}{2} a u^{2}\right\}_{t t}, \quad \\
a= & 4 l_{x}^{2} l_{x x}, \quad b=8 l_{x x}, \quad c=-10 l_{x} l_{x x}, \quad B_{0}=-2 l_{t}, \quad B_{1}=-l_{x}^{4}, \quad B_{2}=4 l_{x}^{3}, \quad B_{3}=-6 l_{x}^{2}, \quad B_{4}=4 l_{x} .
\end{aligned}
$$


Remark 2.2. The key points in the proof of Proposition 2.1 are suitable grouping and the choice of $a, b$ and $c$. Proof. From the assumptions of $\lambda, \mu, \varphi$ and $l$, we can obtain that

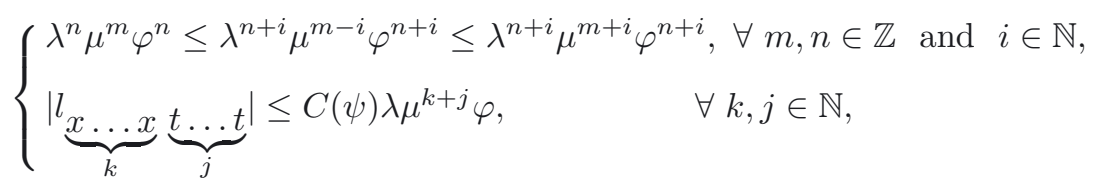

where $\underbrace{l_{k} \ldots x}_{k} \underbrace{t \ldots t}_{j}=\frac{\partial^{k+j} l}{\partial^{k} x \partial^{j} t}$.

Direct computation shows that

$$
\theta\left(y_{t t}-y_{x x x x}\right)=u_{t t}+A_{0} u_{t}+A_{1} u+A_{2} u_{x}+A_{3} u_{x x}+A_{4} u_{x x x}-u_{x x x x}
$$

where

$$
\begin{aligned}
& A_{0}=-2 l_{t}, \\
& A_{1}=l_{t}^{2}-l_{t t}-l_{x}^{4}-4 l_{x} l_{x x x}+l_{x x x x}+6 l_{x}^{2} l_{x x}-3 l_{x x}^{2}, \\
& A_{2}=-12 l_{x} l_{x x}+4 l_{x}^{3}+4 l_{x x x}, \\
& A_{3}=-6 l_{x}^{2}+6 l_{x x}, \\
& A_{4}=4 l_{x} .
\end{aligned}
$$

Set

$$
\begin{aligned}
& I_{1}=-u_{x x x x}+B_{1} u+B_{3} u_{x x}+u_{t t}+c u_{x}, \\
& I_{2}=B_{2} u_{x}+B_{4} u_{x x x}+B_{0} u_{t}+a u+b u_{x x}, \\
& R=\theta\left(y_{t t}-y_{x x x x}\right)-I_{1}-I_{2}=S_{0} u+S_{1} u_{x}+S_{2} u_{x x},
\end{aligned}
$$

where

$$
\begin{aligned}
& S_{0}=l_{t}^{2}-l_{t t}-4 l_{x} l_{x x x}+l_{x x x x}-3 l_{x x}^{2}+2 l_{x}^{2} l_{x x}, \\
& S_{1}=4 l_{x x x}-2 l_{x} l_{x x}, S_{2}=-2 l_{x x} .
\end{aligned}
$$

Step 1. We shall prove the following equality

$$
\begin{aligned}
I_{1} \cdot I_{2} & =u^{2}\{\ldots\}+u_{x}^{2}\{\ldots\}+u_{x x}^{2}\{\ldots\}+u_{x x x}^{2}\{\ldots\}+u_{t}^{2}\{\ldots\}+u_{x t}^{2}\{\ldots\}+u_{x t} u_{x x x}\{\ldots\} \\
& +u_{t} u_{x}\{\ldots\}+u_{t} u_{x x x}\{\ldots\}+\{\ldots\}_{x}+\{\ldots\}_{x x}+\{\ldots\}_{x x x}+\{\ldots\}_{x x x x}+\{\ldots\}_{t}+\{\ldots\}_{t t},
\end{aligned}
$$

where

$$
\begin{aligned}
u^{2}\{\ldots\} & =u^{2}\left\{-\frac{1}{2} a_{x x x x}-\frac{1}{2}\left(B_{1} B_{2}\right)_{x}-\frac{1}{2}\left(B_{1} B_{4}\right)_{x x x}+\frac{1}{2}\left(b B_{1}\right)_{x x}-\frac{1}{2}\left(B_{0} B_{1}\right)_{t}+\frac{1}{2}\left(a B_{3}\right)_{x x}+\frac{1}{2} a_{t t}+a B_{1}-\frac{1}{2}(a c)_{x}\right\}, \\
u_{x}^{2}\{\ldots\} & =u_{x}^{2}\left\{\frac{1}{2} B_{2 x x x}+2 a_{x x}+\frac{3}{2}\left(B_{1} B_{4}\right)_{x}-b B_{1}-\frac{1}{2}\left(B_{2} B_{3}\right)_{x}-a B_{3}+\frac{1}{2}\left(B_{0} B_{3}\right)_{t}-\frac{1}{2} b_{t t}+B_{2} c+\frac{1}{2}\left(B_{4} c\right)_{x x}-\frac{1}{2}(b c)_{x}\right\}, \\
u_{x x}^{2}\{\ldots\} & =u_{x x}^{2}\left\{-\frac{3}{2} B_{2 x}-a-\frac{1}{2} b_{x x}-\frac{1}{2}\left(B_{3} B_{4}\right)_{x}+b B_{3}-B_{4} c\right\} \\
u_{x x x}^{2}\{\ldots\} & =u_{x x x}^{2}\left\{\frac{1}{2} B_{4 x}+b\right\} \\
u_{t}^{2}\{\ldots\} & =u_{t}^{2}\left\{\frac{1}{2} B_{2 x}-\frac{1}{2} B_{0 t}-a+\frac{1}{2} B_{4 x x x}-\frac{1}{2} b_{x x}\right\}, \\
u_{x t}^{2}\{\ldots\} & =u_{x t}^{2}\left\{-\frac{3}{2} B_{4 x}+b\right\} \\
u_{x t} u_{x x x}\{\ldots\} & =u_{x t} u_{x x x}\left\{B_{0}\right\} \\
u_{t} u_{x}\{\ldots\} & =u_{t} u_{x}\left\{-\left(B_{0} B_{3}\right)_{x}-B_{2 t}+b_{x t}+B_{0} c\right\} \\
u_{t} u_{x x x}\{\ldots\} & =u_{t} u_{x x x}\left\{B_{0 x}-B_{4 t}\right\}
\end{aligned}
$$


and $\{\ldots\}_{x},\{\ldots\}_{x x},\{\ldots\}_{x x x},\{\ldots\}_{x x x x},\{\ldots\}_{t},\{\ldots\}_{t t}$ are the same as in Proposition 2.1.

Indeed, (2.3) can be obtained from the following equations

$$
\begin{aligned}
& u_{x x x x} B_{2} u_{x}=\frac{1}{2}\left(B_{2} u_{x}^{2}\right)_{x x x}-\frac{3}{2}\left(B_{2 x} u_{x}^{2}\right)_{x x}+\frac{3}{2}\left(B_{2 x x} u_{x}^{2}-B_{2} u_{x x}^{2}\right)_{x}+\frac{3}{2} B_{2 x} u_{x x}^{2}-\frac{1}{2} B_{2 x x x} u_{x}^{2}, \\
& u_{x x x x} B_{4} u_{x x x}=\frac{1}{2}\left(\left(B_{4} u_{x x x}^{2}\right)_{x}-B_{4 x} u_{x x x}^{2}\right) \text {, } \\
& u_{x x x x} a u=\frac{1}{2}\left(a u^{2}\right)_{x x x x}-2\left(a_{x} u^{2}\right)_{x x x}+\left(3 a_{x x} u^{2}-2 a u_{x}^{2}\right)_{x x} \\
& +\left(4 a_{x} u_{x}^{2}-2 a_{x x x} u^{2}\right)_{x}+a u_{x x}^{2}-2 a_{x x} u_{x}^{2}+\frac{1}{2} a_{x x x x} u^{2}, \\
& u_{x x x x} b u_{x x}=\frac{1}{2}\left(b u_{x x}^{2}\right)_{x x}-\left(b_{x} u_{x x}^{2}\right)_{x}-b u_{x x x}^{2}+\frac{1}{2} b_{x x} u_{x x}^{2}, \\
& u_{x x x x} B_{0} u_{t}=\left(B_{0} u_{t} u_{x x x}\right)_{x}-B_{0 x} u_{t} u_{x x x}-B_{0} u_{x t} u_{x x x}, \\
& B_{1} u B_{2} u_{x}=\frac{1}{2}\left(\left(B_{1} B_{2} u^{2}\right)_{x}-\left(B_{1} B_{2}\right)_{x} u^{2}\right), \\
& B_{1} u B_{4} u_{x x x}=\frac{1}{2}\left(B_{1} B_{4} u^{2}\right)_{x x x}-\frac{3}{2}\left(\left(B_{1} B_{4}\right)_{x} u^{2}\right)_{x x}+\frac{3}{2}\left(B_{1} B_{4}\right)_{x} u_{x}^{2} \\
& +\frac{3}{2}\left(\left(B_{1} B_{4}\right)_{x x} u^{2}-B_{1} B_{4} u_{x}^{2}\right)_{x}-\frac{1}{2}\left(B_{1} B_{4}\right)_{x x x} u^{2}, \\
& B_{1} u b u_{x x}=\frac{1}{2}\left(b B_{1} u^{2}\right)_{x x}-\left[\left(b B_{1}\right)_{x} u^{2}\right]_{x}-b B_{1} u_{x}^{2}+\frac{1}{2}\left(b B_{1}\right)_{x x} u^{2}, \\
& B_{1} u B_{0} u_{t}=\frac{1}{2}\left(\left(B_{0} B_{1} u^{2}\right)_{t}-\left(B_{0} B_{1}\right)_{t} u^{2}\right), \\
& B_{3} u_{x x} B_{2} u_{x}=\frac{1}{2}\left(\left(B_{2} B_{3} u_{x}^{2}\right)_{x}-\left(B_{2} B_{3}\right)_{x} u_{x}^{2}\right), \\
& B_{3} u_{x x} B_{4} u_{x x x}=\frac{1}{2}\left(\left(B_{3} B_{4} u_{x x}^{2}\right)_{x}-\left(B_{3} B_{4}\right)_{x} u_{x x}^{2}\right), \\
& B_{3} u_{x x} a u=\frac{1}{2}\left(a B_{3} u^{2}\right)_{x x}-\left(\left(a B_{3}\right)_{x} u^{2}\right)_{x}-a B_{3} u_{x}^{2}+\frac{1}{2}\left(a B_{3}\right)_{x x} u^{2}, \\
& B_{3} u_{x x} B_{0} u_{t}=\frac{1}{2}\left(B_{0} B_{3}\right)_{t} u_{x}^{2}-\left(B_{0} B_{3}\right)_{x} u_{t} u_{x}-\frac{1}{2}\left(B_{0} B_{3} u_{x}^{2}\right)_{t}+\left(B_{0} B_{3} u_{x} u_{t}\right)_{x} \text {, } \\
& u_{t t} B_{2} u_{x}=\left(B_{2} u_{t} u_{x}\right)_{t}-B_{2 t} u_{t} u_{x}-\frac{1}{2}\left(\left(B_{2} u_{t}^{2}\right)_{x}-B_{2 x} u_{t}^{2}\right), \\
& u_{t t} B_{0} u_{t}=\frac{1}{2}\left(\left(B_{0} u_{t}^{2}\right)_{t}-B_{0 t} u_{t}^{2}\right) \text {, } \\
& u_{t t} a u=\frac{1}{2}\left(a u^{2}\right)_{t t}-\left(a_{t} u^{2}\right)_{t}-a u_{t}^{2}+\frac{1}{2} a_{t t} u^{2}, \\
& u_{t t} B_{4} u_{x x x}=\left(B_{4} u_{t} u_{x x x}\right)_{t}-B_{4 t} u_{t} u_{x x x}-\frac{1}{2}\left(B_{4} u_{t}^{2}\right)_{x x x}+\frac{3}{2}\left(B_{4 x} u_{t}^{2}\right)_{x x} \\
& -\frac{3}{2}\left(B_{4 x x} u_{t}^{2}-B_{4} u_{x t}^{2}\right)_{x}-\frac{3}{2} B_{4 x} u_{x t}^{2}+\frac{1}{2} B_{4 x x x} u_{t}^{2}, \\
& u_{t t} b u_{x x}=\left(b u_{t} u_{x x}\right)_{t}+b_{x t} u_{t} u_{x}-\frac{1}{2} b_{t t} u_{x}^{2}+\frac{1}{2}\left(b_{t} u_{x}^{2}\right)_{t}-\left(b_{t} u_{t} u_{x}\right)_{x}-\frac{1}{2}\left(b u_{t}^{2}\right)_{x x}+\left(b_{x} u_{t}^{2}\right)_{x}+b u_{x t}^{2}-\frac{1}{2} b_{x x} u_{t}^{2} \text {, } \\
& B_{4} u_{x x x} c u_{x}=\frac{1}{2}\left(B_{4} c u_{x}^{2}\right)_{x x}-\left(\left(B_{4} c\right)_{x} u_{x}^{2}\right)_{x}-B_{4} c u_{x x}^{2}+\frac{1}{2}\left(B_{4} c\right)_{x x} u_{x}^{2} \\
& \text { aucu }_{x}=\frac{1}{2}\left(\left(a c u^{2}\right)_{x}-(a c)_{x} u^{2}\right), \\
& b u_{x x} c u_{x}=\frac{1}{2}\left(\left(b c u_{x}^{2}\right)_{x}-(b c)_{x} u_{x}^{2}\right) \text {. }
\end{aligned}
$$


Step 2. We shall prove estimate (2.1).

Indeed,

$$
\begin{aligned}
u^{2}\{\ldots\} & =u^{2}\left\{10 \lambda^{7} \mu^{8} \varphi^{7} \psi_{x}^{8}+R_{0}\right\}, \\
u_{x}^{2}\{\ldots\} & =u_{x}^{2}\left\{22 \lambda^{5} \mu^{6} \varphi^{5} \psi_{x}^{6}+R_{1}\right\}, \\
u_{x x}^{2}\{\ldots\} & =u_{x x}^{2}\left\{6 \lambda^{3} \mu^{4} \varphi^{3} \psi_{x}^{4}+R_{2}\right\}, \\
u_{x x x}^{2}\{\ldots\} & =u_{x x x}^{2}\left\{10 \lambda \mu^{2} \varphi \psi_{x}^{2}+R_{3}\right\}, \\
u_{t}^{2}\{\ldots\} & =u_{t}^{2}\left\{2 \lambda^{3} \mu^{4} \varphi^{3} \psi_{x}^{4}+R_{4}\right\}, \\
u_{x t}^{2}\{\ldots\} & =u_{x t}^{2}\left\{2 l_{x x}\right\}, \\
u_{x t} u_{x x x}\{\ldots\} & =u_{x t} u_{x x x}\left\{2 l_{t}\right\}, \\
u_{t} u_{x}\{\ldots\} & =u_{t} u_{x}\left\{-24 l_{x x} l_{x}^{2}-4 l_{t} l_{x} l_{x x}+8 l_{x x x t}\right\}, \\
u_{t} u_{x x x}\{\ldots\} & =u_{t} u_{x x x}\left\{-6 l_{x t}\right\},
\end{aligned}
$$

where

$$
\begin{aligned}
& \left|R_{0}\right| \leq C \lambda^{7} \mu^{7} \varphi^{7}, \quad\left|R_{1}\right| \leq C \lambda^{5} \mu^{5} \varphi^{5}, \quad\left|R_{2}\right| \leq C \lambda^{3} \mu^{3} \varphi^{3}, \\
& \left|R_{3}\right| \leq C \lambda \mu \varphi, \quad\left|R_{4}\right| \leq C \lambda^{3} \mu^{3} \varphi^{3} .
\end{aligned}
$$

Here we have used (2.2). Similarly, we have

$$
\begin{aligned}
\left|u_{x t} u_{x x x}\{\ldots\}\right| & \leq C\left(\lambda \mu \varphi u_{x x x}^{2}+\lambda \mu \varphi u_{x t}^{2}\right), \\
\left|u_{t} u_{x}\{\ldots\}\right| & \leq C\left(\lambda^{5} \mu^{5} \varphi^{5} u_{x}^{2}+\lambda^{3} \mu^{3} \varphi^{3} u_{t}^{2}\right), \\
\left|u_{t} u_{x x x}\{\ldots\}\right| & \leq C\left(\lambda \mu \varphi u_{x x x}^{2}+\lambda^{3} \mu^{3} \varphi^{3} u_{t}^{2}\right), \\
S_{0}^{2} u^{2} & \leq C \lambda^{7} \mu^{7} \varphi^{7} u^{2} \\
S_{1}^{2} u_{x}^{2} & \leq C \lambda^{5} \mu^{5} \varphi^{5} u_{x}^{2} \\
S_{2}^{2} u_{x x}^{2} & \leq C \lambda^{3} \mu^{3} \varphi^{3} u_{x x}^{2}
\end{aligned}
$$

this implies

$$
\begin{aligned}
I_{1} I_{2} & \leq \frac{1}{2}\left(I_{1}+I_{2}\right)^{2} \\
& =\frac{1}{2}\left[\theta\left(y_{t t}-y_{x x x x}\right)-R\right]^{2} \\
& \leq \theta^{2}\left|y_{t t}-y_{x x x x}\right|^{2}+|R|^{2} \\
& \leq C\left(\theta^{2}\left|y_{t t}-y_{x x x x}\right|^{2}+S_{0}^{2} u^{2}+S_{1}^{2} u_{x}^{2}+S_{2}^{2} u_{x x}^{2}\right) \\
& \leq C\left(\theta^{2}\left|y_{t t}-y_{x x x x}\right|^{2}+\lambda^{7} \mu^{7} \varphi^{7} u^{2}+\lambda^{5} \mu^{5} \varphi^{5} u_{x}^{2}+\lambda^{3} \mu^{3} \varphi^{3} u_{x x}^{2}\right) .
\end{aligned}
$$

Thanks to (2.4)-(2.7) all together with (2.3), we can obtain (2.1).

\section{Proof of Theorem 1.1}

Through this section, we make the following assumptions:

(A1) Let $T_{0}, T^{\prime}, T^{\prime \prime}, \gamma, b_{0}$ satisfy

$$
\left\{\begin{array}{l}
0<\gamma<T^{\prime}<\frac{T_{0}}{2}<T^{\prime \prime}<T_{0}-\gamma \\
\left|\frac{T_{0}}{2}-T^{\prime}\right|=\left|T^{\prime \prime}-\frac{T_{0}}{2}\right| \\
\max \left\{T^{\prime \prime}-\frac{T_{0}}{2},\left(1+\left(\frac{T_{0}}{2}-T^{\prime}\right)^{2}\right)^{\frac{1}{2}}\right\}<b_{0}<\frac{T_{0}}{2}-\gamma .
\end{array}\right.
$$


(A2) For a measurable $F \subset Q_{0} \triangleq I \times\left(0, T_{0}\right)$ and a function $g$ satisfies $g, g_{x}, g_{x x}, g_{x x x}, g_{t}, g_{x t} \in L^{2}(F)$, we set

$$
\|g\|_{\star, F}=\left(\|g\|_{L^{2}(F)}^{2}+\left\|g_{x}\right\|_{L^{2}(F)}^{2}+\left\|g_{x x}\right\|_{L^{2}(F)}^{2}+\left\|g_{x x x}\right\|_{L^{2}(F)}^{2}+\left\|g_{t}\right\|_{L^{2}(F)}^{2}+\left\|g_{x t}\right\|_{L^{2}(F)}^{2}\right)^{\frac{1}{2}} .
$$

(A3) Define $\mathcal{G} \triangleq\left\{g \in L^{2}\left(Q_{0}\right) \mid g_{x}, g_{x x}, g_{x x x}, g_{x x x x}, g_{t}, g_{x t} \in L^{2}\left(Q_{0}\right)\right\}$.

Remark 3.1. The existence of $T_{0}, T^{\prime}, T^{\prime \prime}, \gamma$ and $b_{0}$ is easy to obtain, for example, $T_{0}=4, T^{\prime}=1, T^{\prime \prime}=3, \gamma=$ $0.5, b_{0}=1.42$ satisfies $(\mathrm{A} 1)$.

We borrow some idea from [11].

In order to prove Theorem 1.1, we investigate the following system

$$
\begin{cases}y_{t t}-y_{x x x x}=0 & \text { in } Q_{0}, \\ y(0, t)=0=y(1, t) & \text { in }\left(0, T_{0}\right), \\ y_{x}(0, t)=0=y_{x}(1, t) & \text { in }\left(0, T_{0}\right) .\end{cases}
$$

First, we establish two important lemmas.

Lemma 3.2. There exists a constant $\alpha_{1} \in(0,1)$ such that we have the following estimate

$$
\|y\|_{L^{2}\left(I \times\left(T^{\prime}, T^{\prime \prime}\right)\right)} \leq C\|y\|_{\star, \omega \times\left(\gamma, T_{0}-\gamma\right)}^{\alpha_{1}}\|y\|_{\star, Q_{0}}^{1-\alpha_{1}},
$$

where $y \in \mathcal{G}$ solves (3.1).

Proof. Introduce $\phi \in C_{0}^{\infty}\left(\gamma, T_{0}-\gamma\right)$ and $\psi_{0} \in C^{\infty}(\bar{I})$ which enjoy the following properties

$$
\begin{cases}0 \leq \phi(t) \leq 1 & t \in\left(\gamma, T_{0}-\gamma\right) \\ \phi(t)=1 & t \in\left(\frac{T_{0}}{2}-b_{0}, \frac{T_{0}}{2}+b_{0}\right) \\ \psi_{0}(x)>0 & x \in I, \\ \left|\psi_{0 x}\right|>0 & x \in \bar{I} \backslash \omega, \\ \psi_{0}(0)=\psi_{0}(1)=0, & \psi_{0 x}(0)>0, \quad \psi_{0 x}(1)<0, \quad\left\|\psi_{0}\right\|_{C(\bar{I})}=1 .\end{cases}
$$

The existence of $\psi_{0}$ can be found in [15].

Set $\psi_{1}(x, t)=\psi_{0}(x)-\left(t-\frac{T_{0}}{2}\right)^{2}+\widetilde{C}_{1}$, where $\widetilde{C}_{1}$ is chosen to be so large to make $\psi_{1}>0$. According to (A1), it is obvious that $\left(T^{\prime}, T^{\prime \prime}\right) \subset\left(\frac{T_{0}}{2}-b_{0}, \frac{T_{0}}{2}+b_{0}\right)$.

We here let $\psi(x, t)=\psi_{1}(x, t)$ in Proposition 2.1 and apply (2.1) to $\bar{y}=\phi y$ and $w=\theta \bar{y}$, some straightforward calculation gives that

$$
\begin{aligned}
& \int_{Q_{0}}\left(\lambda^{7} \mu^{8} \varphi^{7} \psi_{1 x}^{8} w^{2}+\lambda^{5} \mu^{6} \varphi^{5} \psi_{1 x}^{6} w_{x}^{2}+\lambda^{3} \mu^{4} \varphi^{3} \psi_{1 x}^{4} w_{x x}^{2}\right.+\lambda \mu^{2} \varphi \psi_{1 x}^{2} w_{x x x}^{2} \\
&\left.+\lambda^{3} \mu^{4} \varphi^{3} \psi_{1 x}^{4} w_{t}^{2}+\lambda \mu^{2} \varphi \psi_{1 x}^{2} w_{x t}^{2}+\{\ldots\}_{x}+\{\ldots\}_{x x}+\{\ldots\}_{x x x}+\{\ldots\}_{x x x x}+\{\ldots\}_{t}+\{\ldots\}_{t t}\right) \mathrm{d} x \mathrm{~d} t \\
& \leq C(\psi) \int_{Q_{0}}\left(\lambda^{7} \mu^{7} \varphi^{7} w^{2}+\lambda^{5} \mu^{5} \varphi^{5} w_{x}^{2}+\lambda^{3} \mu^{3} \varphi^{3} w_{x x}^{2}+\lambda \mu \varphi w_{x x x}^{2}\right. \\
&\left.+\lambda^{3} \mu^{3} \varphi^{3} w_{t}^{2}+\lambda \mu \varphi w_{x t}^{2}+\theta^{2}\left|\bar{y}_{t t}-\bar{y}_{x x x x}\right|^{2}\right) \mathrm{d} x \mathrm{~d} t .
\end{aligned}
$$

It follows from (3.3) that

$$
w(0, t)=w(1, t)=w_{x}(0, t)=w_{x}(1, t)=0 \quad \forall t \in\left(0, T_{0}\right)
$$


and

$$
w(x, 0)=w_{t}(x, 0)=w\left(x, T_{0}\right)=w_{t}\left(x, T_{0}\right)=0 \quad \forall x \in I .
$$

Thus

$$
\begin{aligned}
\int_{Q_{0}}\left(\{\ldots\}_{t}+\{\ldots\}_{t t}\right) \mathrm{d} x \mathrm{~d} t & =0 \\
\int_{Q_{0}}\left(\{\ldots\}_{x}+\{\ldots\}_{x x}+\{\ldots\}_{x x x}+\{\ldots\}_{x x x x}\right) \mathrm{d} x \mathrm{~d} t & =\int_{0}^{T_{0}}\left(w_{x x}^{2}\left(-10 l_{x}^{3}+4 l_{x x x}\right)-8 l_{x x} w_{x x} w_{x x x}\right. \\
& \left.+w_{x x x}^{2}\left(-2 l_{x}\right)\right)\left.(\cdot, t)\right|_{0} ^{1} \mathrm{~d} t \\
\triangleq & V(1)-V(0) .
\end{aligned}
$$

If we choose $\lambda \geq \lambda_{0}$ with $\lambda_{0}$ large enough and note that $\psi_{0 x}(1)<0, \psi_{0 x}(0)>0$, then it holds that

$$
-V(0) \geq 0, V(1) \geq 0
$$

In view of (3.4)-(3.6), there holds

$$
\begin{gathered}
\int_{Q_{0}}\left(\lambda^{7} \mu^{8} \varphi^{7} \psi_{1 x}^{8} w^{2}+\lambda^{5} \mu^{6} \varphi^{5} \psi_{1 x}^{6} w_{x}^{2}+\lambda^{3} \mu^{4} \varphi^{3} \psi_{1 x}^{4} w_{x x}^{2}+\lambda \mu^{2} \varphi \psi_{1 x}^{2} w_{x x x}^{2}+\lambda^{3} \mu^{4} \varphi^{3} \psi_{1 x}^{4} w_{t}^{2}+\lambda \mu^{2} \varphi \psi_{1 x}^{2} w_{x t}^{2}\right) \mathrm{d} x \mathrm{~d} t \\
\leq C(\psi) \int_{Q_{0}}\left(\lambda^{7} \mu^{7} \varphi^{7} w^{2}+\lambda^{5} \mu^{5} \varphi^{5} w_{x}^{2}+\lambda^{3} \mu^{3} \varphi^{3} w_{x x}^{2}+\lambda \mu \varphi w_{x x x}^{2}\right. \\
\left.+\lambda^{3} \mu^{3} \varphi^{3} w_{t}^{2}+\lambda \mu \varphi w_{x t}^{2}+\theta^{2}\left|\bar{y}_{t t}-\bar{y}_{x x x x}\right|^{2}\right) \mathrm{d} x \mathrm{~d} t
\end{gathered}
$$

Set $Q_{0}^{\omega}=\omega \times\left(0, T_{0}\right)$. Recall that $\left|\psi_{1 x}\right|>0$ in $\bar{I} \backslash \omega$, it follows that

$$
\begin{aligned}
\int_{Q_{0} \backslash Q_{0}^{\omega}}\left(\lambda^{7} \mu^{8} \varphi^{7} w^{2}+\right. & \left.\lambda^{5} \mu^{6} \varphi^{5} w_{x}^{2}+\lambda^{3} \mu^{4} \varphi^{3} w_{x x}^{2}+\lambda \mu^{2} \varphi w_{x x x}^{2}+\lambda^{3} \mu^{4} \varphi^{3} w_{t}^{2}+\lambda \mu^{2} \varphi w_{x t}^{2}\right) \mathrm{d} x \mathrm{~d} t \\
\leq C_{1}(\psi) \int_{Q_{0}}\left(\lambda^{7} \mu^{7} \varphi^{7} w^{2}+\lambda^{5} \mu^{5} \varphi^{5} w_{x}^{2}\right. & +\lambda^{3} \mu^{3} \varphi^{3} w_{x x}^{2}+\lambda \mu \varphi w_{x x x}^{2} \\
& \left.+\lambda^{3} \mu^{3} \varphi^{3} w_{t}^{2}+\lambda \mu \varphi w_{x t}^{2}+\theta^{2}\left|\bar{y}_{t t}-\bar{y}_{x x x x}\right|^{2}\right) \mathrm{d} x \mathrm{~d} t
\end{aligned}
$$

from which if we choose $\mu=\mu_{0}=C_{1}(\psi)+1$, then it holds that

$$
\begin{aligned}
& \int_{Q_{0} \backslash Q_{0}^{\omega}}\left(\lambda^{7} \mu^{7} \varphi^{7} w^{2}+\right.\left.\lambda^{5} \mu^{5} \varphi^{5} w_{x}^{2}+\lambda^{3} \mu^{3} \varphi^{3} w_{x x}^{2}+\lambda \mu \varphi w_{x x x}^{2}+\lambda^{3} \mu^{3} \varphi^{3} w_{t}^{2}+\lambda \mu \varphi w_{x t}^{2}\right) \mathrm{d} x \mathrm{~d} t \\
& \leq C_{2}(\psi)\left(\int_{Q_{0}} \theta^{2}\left|\bar{y}_{t t}-\bar{y}_{x x x x}\right|^{2} \mathrm{~d} x \mathrm{~d} t+\int_{Q_{0}^{\omega}}\left(\lambda^{7} \mu^{7} \varphi^{7} w^{2}+\lambda^{5} \mu^{5} \varphi^{5} w_{x}^{2}\right.\right. \\
&\left.\left.+\lambda^{3} \mu^{3} \varphi^{3} w_{x x}^{2}+\lambda \mu \varphi w_{x x x}^{2}+\lambda^{3} \mu^{3} \varphi^{3} w_{t}^{2}+\lambda \mu \varphi w_{x t}^{2}\right) \mathrm{~d} x \mathrm{~d} t\right) .
\end{aligned}
$$

Then

$$
\begin{gathered}
\int_{Q_{0} \backslash Q_{0}^{\omega}}\left(\lambda^{7} \mu^{7} \varphi^{7} w^{2}+\lambda^{5} \mu^{5} \varphi^{5} w_{x}^{2}+\lambda^{3} \mu^{3} \varphi^{3} w_{x x}^{2}+\lambda \mu \varphi w_{x x x}^{2}+\lambda^{3} \mu^{3} \varphi^{3} w_{t}^{2}+\lambda \mu \varphi w_{x t}^{2}\right) \mathrm{d} x \mathrm{~d} t \\
+\int_{Q_{0}^{\omega}}\left(\lambda^{7} \mu^{7} \varphi^{7} w^{2}+\lambda^{5} \mu^{5} \varphi^{5} w_{x}^{2}+\lambda^{3} \mu^{3} \varphi^{3} w_{x x}^{2}+\lambda \mu \varphi w_{x x x}^{2}+\lambda^{3} \mu^{3} \varphi^{3} w_{t}^{2}+\lambda \mu \varphi w_{x t}^{2}\right) \mathrm{d} x \mathrm{~d} t \\
\leq C\left(\int_{Q_{0}} \theta^{2}\left|\bar{y}_{t t}-\bar{y}_{x x x x}\right|^{2} \mathrm{~d} x \mathrm{~d} t+\int_{Q_{0}^{\omega}}\left(\lambda^{7} \mu^{7} \varphi^{7} w^{2}+\lambda^{5} \mu^{5} \varphi^{5} w_{x}^{2}\right.\right. \\
\left.\left.+\lambda^{3} \mu^{3} \varphi^{3} w_{x x}^{2}+\lambda \mu \varphi w_{x x x}^{2}+\lambda^{3} \mu^{3} \varphi^{3} w_{t}^{2}+\lambda \mu \varphi w_{x t}^{2}\right) \mathrm{~d} x \mathrm{~d} t\right)
\end{gathered}
$$


and thus

$$
\begin{array}{r}
\int_{Q_{0}}\left(\lambda^{7} \mu^{7} \varphi^{7} w^{2}+\lambda^{5} \mu^{5} \varphi^{5} w_{x}^{2}+\lambda^{3} \mu^{3} \varphi^{3} w_{x x}^{2}+\lambda \mu \varphi w_{x x x}^{2}+\lambda^{3} \mu^{3} \varphi^{3} w_{t}^{2}+\lambda \mu \varphi w_{x t}^{2}\right) \mathrm{d} x \mathrm{~d} t \\
\leq C\left(\int_{Q_{0}} \theta^{2}\left|\bar{y}_{t t}-\bar{y}_{x x x x}\right|^{2} \mathrm{~d} x \mathrm{~d} t+\int_{Q_{0}^{\omega}}\left(\lambda^{7} \mu^{7} \varphi^{7} w^{2}+\lambda^{5} \mu^{5} \varphi^{5} w_{x}^{2}\right.\right. \\
\left.\left.+\lambda^{3} \mu^{3} \varphi^{3} w_{x x}^{2}+\lambda \mu \varphi w_{x x x}^{2}+\lambda^{3} \mu^{3} \varphi^{3} w_{t}^{2}+\lambda \mu \varphi w_{x t}^{2}\right) \mathrm{~d} x \mathrm{~d} t\right),
\end{array}
$$

from which it holds that

$$
\begin{aligned}
\int_{Q_{0}}\left(\lambda^{7} \varphi^{7} w^{2}+\lambda^{5} \varphi^{5} w_{x}^{2}\right. & \left.+\lambda^{3} \varphi^{3} w_{x x}^{2}+\lambda \varphi w_{x x x}^{2}+\lambda^{3} \varphi^{3} w_{t}^{2}+\lambda \varphi w_{x t}^{2}\right) \mathrm{d} x \mathrm{~d} t \\
\leq C(\mu)\left(\int_{Q_{0}} \theta^{2}\left|\bar{y}_{t t}-\bar{y}_{x x x x}\right|^{2} \mathrm{~d} x \mathrm{~d} t\right. & +\int_{Q_{0}^{\omega}}\left(\lambda^{7} \varphi^{7} w^{2}+\lambda^{5} \varphi^{5} w_{x}^{2}\right. \\
& \left.\left.+\lambda^{3} \varphi^{3} w_{x x}^{2}+\lambda \varphi w_{x x x}^{2}+\lambda^{3} \varphi^{3} w_{t}^{2}+\lambda \varphi w_{x t}^{2}\right) \mathrm{~d} x \mathrm{~d} t\right) .
\end{aligned}
$$

Returning $w$ to $\theta \bar{y}$ ( $\mu=\mu_{0}$ is now fixed), we can obtain that

$$
\begin{gathered}
\int_{Q_{0}}\left(\lambda^{7} \varphi^{7} \theta^{2} \bar{y}^{2}+\lambda^{5} \varphi^{5} \theta^{2} \bar{y}_{x}^{2}+\lambda^{3} \varphi^{3} \theta^{2} \bar{y}_{x x}^{2}+\lambda \varphi \theta^{2} \bar{y}_{x x x}^{2}+\lambda^{3} \varphi^{3} \theta^{2} \bar{y}_{t}^{2}+\lambda \varphi \theta^{2} \bar{y}_{x t}^{2}\right) \mathrm{d} x \mathrm{~d} t \\
\leq C\left(\int_{Q_{0}} \theta^{2}\left|\bar{y}_{t t}-\bar{y}_{x x x x}\right|^{2} \mathrm{~d} x \mathrm{~d} t+\int_{Q_{0}^{\omega}}\left(\lambda^{7} \varphi^{7} \theta^{2} \bar{y}^{2}+\lambda^{5} \varphi^{5} \theta^{2} \bar{y}_{x}^{2}\right.\right. \\
\left.\left.+\lambda^{3} \varphi^{3} \theta^{2} \bar{y}_{x x}^{2}+\lambda \varphi \theta^{2} \bar{y}_{x x x}^{2}+\lambda^{3} \varphi^{3} \theta^{2} \bar{y}_{t}^{2}+\lambda \varphi \theta^{2} \bar{y}_{x t}^{2}\right) \mathrm{~d} x \mathrm{~d} t\right) .
\end{gathered}
$$

Taking into account the construction of $\phi$ and $\psi_{0}$, we have

$$
\begin{aligned}
& \int_{Q_{0}}\left(\lambda^{7} \varphi^{7} \theta^{2} \bar{y}^{2}+\lambda^{5} \varphi^{5} \theta^{2} \bar{y}_{x}^{2}+\lambda^{3} \varphi^{3} \theta^{2} \bar{y}_{x x}^{2}+\lambda \varphi \theta^{2} \bar{y}_{x x x}^{2}+\lambda^{3} \varphi^{3} \theta^{2} \bar{y}_{t}^{2}+\lambda \varphi \theta^{2} \bar{y}_{x t}^{2}\right) \mathrm{d} x \mathrm{~d} t \\
& \geq \int_{Q_{0}} \lambda^{7} \varphi^{7} \theta^{2} \bar{y}^{2} \\
& \geq \int_{I \times\left(T^{\prime}, T^{\prime \prime}\right)} \lambda^{7} \varphi^{7} \theta^{2} \bar{y}^{2} \\
& \geq \lambda^{7} \mathrm{e}^{7 \mu\left(\widetilde{C}_{1}-\left(\frac{T_{0}}{2}-T^{\prime}\right)^{2}\right)} \mathrm{e}^{2 \lambda \mathrm{e}^{\mu\left(\widetilde{C}_{1}-\left(\frac{T_{0}}{2}-T^{\prime}\right)^{2}\right)}} \int_{I \times\left(T^{\prime}, T^{\prime \prime}\right)} y^{2} \mathrm{~d} x \mathrm{~d} t, \\
& \int_{Q_{0}^{\omega}}\left(\lambda^{7} \varphi^{7} \theta^{2} \bar{y}^{2}+\lambda^{5} \varphi^{5} \theta^{2} \bar{y}_{x}^{2}+\lambda^{3} \varphi^{3} \theta^{2} \bar{y}_{x x}^{2}+\lambda \varphi \theta^{2} \bar{y}_{x x x}^{2}+\lambda^{3} \varphi^{3} \theta^{2} \bar{y}_{t}^{2}+\lambda \varphi \theta^{2} \bar{y}_{x t}^{2}\right) \mathrm{d} x \mathrm{~d} t \\
& \leq C \lambda^{7} \mathrm{e}^{7 \mu\left(\widetilde{C}_{1}+1\right)} \mathrm{e}^{2 \lambda \mathrm{e}^{\mu\left(\widetilde{C}_{1}+1\right)}} \int_{\gamma}^{T_{0}-\gamma} \int_{\omega}\left(y^{2}+y_{x}^{2}+y_{x x}^{2}+y_{x x x}^{2}+y_{t}^{2}+y_{x t}^{2}\right) \mathrm{d} x \mathrm{~d} t \\
& =C \lambda^{7} \mathrm{e}^{7 \mu\left(\widetilde{C}_{1}+1\right)} \mathrm{e}^{2 \lambda \mathrm{e}^{\mu\left(\tilde{C}_{1}+1\right)}}\|y\|_{\star, \omega \times\left(\gamma, T_{0}-\gamma\right)}^{2}
\end{aligned}
$$


and

$$
\begin{aligned}
\int_{Q_{0}} \theta^{2}\left|\bar{y}_{t t}-\bar{y}_{x x x x}\right|^{2} \mathrm{~d} x \mathrm{~d} t & =\int_{Q_{0}} \theta^{2}\left|\phi_{t t} y+2 \phi_{t} y_{t}\right|^{2} \mathrm{~d} x \mathrm{~d} t=\int_{\left(0, T_{0}\right) \backslash\left(\frac{T_{0}}{2}-b_{0}, \frac{T_{0}}{2}+b_{0}\right)} \int_{I} \theta^{2}\left|\phi_{t t} y+2 \phi_{t} y_{t}\right|^{2} \mathrm{~d} x \mathrm{~d} t \\
& \leq C \mathrm{e}^{2 \lambda \mathrm{e}^{\mu\left(1-b_{0}^{2}+\tilde{C}_{1}\right)}}\|y\|_{\star, Q_{0}}^{2} .
\end{aligned}
$$

From the above estimates and (3.8), we know that

$$
\begin{aligned}
\lambda^{7} \mathrm{e}^{7 \mu\left(\widetilde{C}_{1}-\left(\frac{T_{0}}{2}-T^{\prime}\right)^{2}\right)} \mathrm{e}^{2 \lambda \mathrm{e}^{\mu\left(\tilde{C}_{1}-\left(\frac{T_{0}}{2}-T^{\prime}\right)^{2}\right)}} & \|y\|_{L^{2}\left(I \times\left(T^{\prime}, T^{\prime \prime}\right)\right)}^{2} \\
\leq & C\left(\mathrm{e}^{2 \lambda \mathrm{e}^{\mu\left(1-b_{0}^{2}+\widetilde{C}_{1}\right)}}\|y\|_{\star, Q_{0}}^{2}+\lambda^{7} \mathrm{e}^{7 \mu\left(\widetilde{C}_{1}+1\right)} \mathrm{e}^{2 \lambda \mathrm{e}^{\mu\left(\tilde{C}_{1}+1\right)}}\|y\|_{\star, \omega \times\left(\gamma, T_{0}-\gamma\right)}^{2}\right),
\end{aligned}
$$

namely,

$$
\begin{aligned}
\|y\|_{L^{2}\left(I \times\left(T^{\prime}, T^{\prime \prime}\right)\right)}^{2} \leq & C\left(\lambda^{-7} \mathrm{e}^{2 \lambda\left(\mathrm{e}^{\mu\left(1-b_{0}^{2}+\widetilde{C}_{1}\right)}-\mathrm{e}^{\mu\left(\tilde{C}_{1}-\left(\frac{T_{0}}{2}-T^{\prime}\right)^{2}\right)}\right)-7 \mu\left(\widetilde{C}_{1}-\left(\frac{T_{0}}{2}-T^{\prime}\right)^{2}\right)}\|y\|_{\star, Q_{0}}^{2}\right. \\
& \left.+\mathrm{e}^{7 \mu\left(1+\left(\frac{T_{0}}{2}-T^{\prime}\right)^{2}\right)} \mathrm{e}^{2 \lambda\left(\mathrm{e}^{\mu\left(\tilde{C}_{1}+1\right)}-\mathrm{e}^{\mu\left(\tilde{C}_{1}-\left(\frac{T_{0}}{2}-T^{\prime}\right)^{2}\right)}\right.}\|y\|_{\star, \omega \times\left(\gamma, T_{0}-\gamma\right)}^{2}\right)
\end{aligned}
$$

Set

$$
\left\{\begin{array}{l}
\varepsilon=\lambda^{-7} \mathrm{e}^{2 \lambda\left(\mathrm{e}^{\mu\left(1-b_{0}^{2}+\tilde{C}_{1}\right)}-\mathrm{e}^{\mu\left(\widetilde{C}_{1}-\left(\frac{T_{0}}{2}-T^{\prime}\right)^{2}\right)}\right)-7 \mu\left(\widetilde{C}_{1}-\left(\frac{T_{0}}{2}-T^{\prime}\right)^{2}\right)}, \\
\varepsilon_{0}=\lambda_{0}^{-7} \mathrm{e}^{2 \lambda_{0}\left(\mathrm{e}^{\mu\left(1-b_{0}^{2}+\tilde{C}_{1}\right)}-\mathrm{e}^{\mu\left(\widetilde{C}_{1}-\left(\frac{T_{0}}{2}-T^{\prime}\right)^{2}\right)}\right)-7 \mu\left(\widetilde{C}_{1}-\left(\frac{T_{0}}{2}-T^{\prime}\right)^{2}\right)}, \\
k=\frac{\mathrm{e}^{\mu\left(\tilde{C}_{1}+1\right)}-\mathrm{e}^{\mu\left(\widetilde{C}_{1}-\left(\frac{T_{0}}{2}-T^{\prime}\right)^{2}\right)}}{\mathrm{e}^{\mu\left(\widetilde{C}_{1}-\left(\frac{T_{0}}{2}-T^{\prime}\right)^{2}\right)}-\mathrm{e}^{\mu\left(1-b_{0}^{2}+\tilde{C}_{1}\right)}},
\end{array}\right.
$$

where $\mu=\mu_{0}$.

(3.9) implies that for any $\varepsilon \in\left(0, \varepsilon_{0}\right]$ the following inequality holds:

$$
\|y\|_{L^{2}\left(I \times\left(T^{\prime}, T^{\prime \prime}\right)\right)} \leq C\left(\varepsilon^{-k}\|y\|_{\star, \omega \times\left(\gamma, T_{0}-\gamma\right)}+\varepsilon\|y\|_{\star, Q_{0}}\right) .
$$

Therefore, (3.10) holds for all $\varepsilon>0$. Further, if we let

$$
\alpha_{1}=\frac{1}{1+k}, \quad \varepsilon=\left(\frac{\|y\|_{\star, \omega \times\left(\frac{T_{0}}{2}-b, \frac{T_{0}}{2}+b\right)}}{\|y\|_{\star, Q_{0}}}\right)^{\alpha_{1}},
$$

(3.10) implies (3.2).

Lemma 3.3. There exists a constant $\alpha_{2} \in(0,1)$ such that we have the following estimate

$$
\|y\|_{\star, \omega \times\left(\gamma, T_{0}-\gamma\right)} \leq C\left(\left\|y_{t}(0)\right\|_{L^{2}(\omega)}+\|y(0)\|_{H^{3}(\omega)}\right)^{\alpha_{2}}\|y\|_{\star, Q_{0}}^{1-\alpha_{2}},
$$

where $y \in \mathcal{G}$ solves (3.1). 
Proof. Since $\omega$ is a nonempty open set, there exists an interval $\omega_{0} \subset \omega$. Set $a=\inf \omega_{0}, b=\sup \omega_{0}$. We introduce the set

$$
N(\tau)=\left\{(x, t) \mid x \in(a+\tau, b-\tau), t \in\left(0, T_{0}-\tau\right)\right\} .
$$

Let $\tau_{i}(i=1,2,3)$ be such that $0<\tau_{3}<\tau_{2}<\tau_{1}<\min \left\{\frac{b-a}{2}, T_{0}\right\}$, thus $N\left(\tau_{1}\right) \subset N\left(\tau_{2}\right) \subset N\left(\tau_{3}\right) \subset \omega_{0} \times\left(0, T_{0}\right)$. We take a function $h=h(x)$ such that

$$
h(x)= \begin{cases}\frac{2}{b-a}\left(x-\frac{a+b}{2}\right)+1 & x \in\left(a, \frac{a+b}{2}\right), \\ \frac{2}{a-b}\left(x-\frac{a+b}{2}\right)+1 & x \in\left[\frac{a+b}{2}, b\right) .\end{cases}
$$

Let $\chi \in C^{\infty}\left(N\left(\tau_{3}\right)\right)$ with the properties

$$
\begin{cases}0 \leq \chi \leq 1 & (x, t) \in N\left(\tau_{3}\right) \\ \chi \equiv 1 & (x, t) \in N\left(\tau_{2}\right) \\ \chi \equiv 0 & (x, t) \in N\left(\tau_{3}\right) \cap U\left(\partial N\left(\tau_{3}\right) \backslash \omega_{0} \times\{0\}\right)\end{cases}
$$

where $U\left(\partial N\left(\tau_{3}\right) \backslash \omega_{0} \times\{0\}\right)$ is a neighborhood of $\partial N\left(\tau_{3}\right) \backslash \omega_{0} \times\{0\}$, which is very small.

Step 1. We shall prove that there exists a constant $\beta_{1} \in(0,1)$ such that

$$
\|y\|_{\star, N\left(\tau_{1}\right)} \leq C\left(\left\|y_{t}(0)\right\|_{L^{2}(\omega)}+\|y(0)\|_{H^{3}(\omega)}\right)^{\beta_{1}}\|y\|_{\star, Q_{0}}^{1-\beta_{1}} .
$$

In order to prove (3.13), set $\psi_{2}(x, t)=h(x)$. We here let $\psi(x, t)=\psi_{2}(x, t)$ in Proposition 2.1. Since $h$ is piecewise smooth in $N\left(\tau_{3}\right)$, i.e. $h$ is smooth in $\left(a+\tau_{3}, \frac{a+b}{2}\right) \times\left(0, T_{0}-\tau_{3}\right)$ and $\left(\frac{a+b}{2}, b-\tau_{3}\right) \times\left(0, T_{0}-\tau_{3}\right)$, we can apply $(2.1)$ to $\tilde{y}=\chi y$ and $u=\theta \tilde{y}$ in $\left(a+\tau_{3}, \frac{a+b}{2}\right) \times\left(0, T_{0}-\tau_{3}\right)$ and $\left(\frac{a+b}{2}, b-\tau_{3}\right) \times\left(0, T_{0}-\tau_{3}\right)$, respectively and then add the two estimates together to obtain that

$$
\begin{aligned}
& \int_{N\left(\tau_{3}\right)}\left(\lambda^{7} \mu^{8} \varphi^{7} u^{2}+\lambda^{5} \mu^{6} \varphi^{5} u_{x}^{2}+\lambda^{3} \mu^{4} \varphi^{3} u_{x x}^{2}+\lambda \mu^{2} \varphi u_{x x x}^{2}+\lambda^{3} \mu^{4} \varphi^{3} u_{t}^{2}+\lambda \mu^{2} \varphi u_{x t}^{2}\right) \mathrm{d} x \mathrm{~d} t \\
& \quad \leq C \int_{N\left(\tau_{3}\right)}\left(\theta^{2}\left|\tilde{y}_{t t}-\tilde{y}_{x x x x}\right|^{2}-\{\ldots\}_{x}-\{\ldots\}_{x x}-\{\ldots\}_{x x x}-\{\ldots\}_{x x x x}-\{\ldots\}_{t}-\{\ldots\}_{t t}\right) \mathrm{d} x \mathrm{~d} t
\end{aligned}
$$

where $\mu$ is large enough with respect to $C\left(\psi_{2}\right)$.

Taking into the construction of $\psi_{2}$ and $\chi$, we have

$$
\begin{aligned}
\int_{N\left(\tau_{3}\right)} & \left(\lambda^{7} \mu^{8} \varphi^{7} u^{2}+\lambda^{5} \mu^{6} \varphi^{5} u_{x}^{2}+\lambda^{3} \mu^{4} \varphi^{3} u_{x x}^{2}+\lambda \mu^{2} \varphi u_{x x x}^{2}+\lambda^{3} \mu^{4} \varphi^{3} u_{t}^{2}+\lambda \mu^{2} \varphi u_{x t}^{2}\right) \mathrm{d} x \mathrm{~d} t \\
& \geq \int_{N\left(\tau_{1}\right)}\left(\lambda^{7} \mu^{8} \varphi^{7} u^{2}+\lambda^{5} \mu^{6} \varphi^{5} u_{x}^{2}+\lambda^{3} \mu^{4} \varphi^{3} u_{x x}^{2}+\lambda \mu^{2} \varphi u_{x x x}^{2}+\lambda^{3} \mu^{4} \varphi^{3} u_{t}^{2}+\lambda \mu^{2} \varphi u_{x t}^{2}\right) \mathrm{d} x \mathrm{~d} t \\
& \geq C \int_{N\left(\tau_{1}\right)}\left(\lambda^{7} \mu^{8} \varphi^{7} \theta^{2} \tilde{y}^{2}+\lambda^{5} \mu^{6} \varphi^{5} \theta^{2} \tilde{y}_{x}^{2}+\lambda^{3} \mu^{4} \varphi^{3} \theta^{2} \tilde{y}_{x x}^{2}+\lambda \mu^{2} \varphi \theta^{2} \tilde{y}_{x x x}^{2}+\lambda^{3} \mu^{4} \varphi^{3} \theta^{2} \tilde{y}_{t}^{2}+\lambda \mu^{2} \varphi \theta^{2} \tilde{y}_{x t}^{2}\right) \mathrm{d} x \mathrm{~d} t \\
& =C \int_{N\left(\tau_{1}\right)}\left(\lambda^{7} \mu^{8} \varphi^{7} \theta^{2} y^{2}+\lambda^{5} \mu^{6} \varphi^{5} \theta^{2} y_{x}^{2}+\lambda^{3} \mu^{4} \varphi^{3} \theta^{2} y_{x x}^{2}+\lambda \mu^{2} \varphi \theta^{2} y_{x x x}^{2}+\lambda^{3} \mu^{4} \varphi^{3} \theta^{2} y_{t}^{2}+\lambda \mu^{2} \varphi \theta^{2} y_{x t}^{2}\right) \mathrm{d} x \mathrm{~d} t \\
& \geq C \int_{N\left(\tau_{1}\right)} \lambda \mu^{2} \varphi \theta^{2}\left(y^{2}+y_{x}^{2}+y_{x x}^{2}+y_{x x x}^{2}+y_{t}^{2}+y_{x t}^{2}\right) \mathrm{d} x \mathrm{~d} t \\
& \geq C \lambda \mu^{2} \mathrm{e}^{h\left(a+\tau_{1}\right) \mu} \mathrm{e}^{2 \lambda \mathrm{e}^{h\left(a+\tau_{1}\right) \mu}}\|y\|_{\star, N\left(\tau_{1}\right)}^{2}
\end{aligned}
$$


and

$$
\begin{aligned}
\int_{N\left(\tau_{3}\right)}( & \left.\{\ldots\}_{x}-\{\ldots\}_{x x}-\{\ldots\}_{x x x}-\{\ldots\}_{x x x x}-\{\ldots\}_{t}-\{\ldots\}_{t t}\right) \mathrm{d} x \mathrm{~d} t \\
= & \int_{N\left(\tau_{3}\right)}\left(-\{\ldots\}_{t}-\{\ldots\}_{t t}\right) \mathrm{d} x \mathrm{~d} t \\
= & \int_{a+\tau_{3}}^{b-\tau_{3}}\left(\left(B_{2} u_{t} u_{x}\right)(x, 0)+\left(B_{4} u_{t} u_{x x x}\right)(x, 0)+\left(b u_{t} u_{x x}\right)(x, 0)+\left(a u u_{t}\right)(x, 0)\right) \mathrm{d} x \\
\leq & C \int_{\omega_{0}}\left(\lambda^{3} \mu^{3}\left(\varphi^{3} u_{t}^{2}\right)(x, 0)+\lambda^{3} \mu^{3}\left(\varphi^{3} u_{x}^{2}\right)(x, 0)+\lambda^{-1} \mu^{-1}\left(\varphi^{-1} u_{x x x}^{2}\right)(x, 0)\right. \\
& \left.+\lambda^{-1} \mu\left(\varphi^{-1} u_{x x}^{2}\right)(x, 0)+\lambda^{3} \mu^{5}\left(\varphi^{3} u^{2}\right)(x, 0)\right) \mathrm{d} x \\
\leq & C \lambda^{3} \mu^{5} \mathrm{e}^{3 \mu} \mathrm{e}^{2 \lambda \mathrm{e}^{\mu}} \int_{\omega_{0}}\left(y_{t}^{2}(x, 0)+y_{x}^{2}(x, 0)+y_{x x x}^{2}(x, 0)+y_{x x}^{2}(x, 0)+y^{2}(x, 0)\right) \mathrm{d} x \\
\leq & C \lambda^{3} \mu^{5} \mathrm{e}^{3 \mu} \mathrm{e}^{2 \lambda \mathrm{e}^{\mu}}\left(\left\|y_{t}(0)\right\|_{L^{2}(\omega)}^{2}+\|y(0)\|_{L^{2}(\omega)}^{2}+\left\|y_{x}(0)\right\|_{L^{2}(\omega)}^{2}+\left\|y_{x x}(0)\right\|_{L^{2}(\omega)}^{2}+\left\|y_{x x x}(0)\right\|_{L^{2}(\omega)}^{2}\right) \\
= & C \lambda^{3} \mu^{5} \mathrm{e}^{3 \mu} \mathrm{e}^{2 \lambda \mathrm{e}^{\mu}}\left(\left\|y_{t}(0)\right\|_{L^{2}(\omega)}^{2}+\|y(0)\|_{H^{3}(\omega)}^{2}\right) .
\end{aligned}
$$

Noting that $\tilde{y}$ solves

$$
\tilde{y}_{t t}-\tilde{y}_{x x x x}=y\left(\chi_{t t}-\chi_{x x x x}\right)+2 y_{t} \chi_{t}-4 y_{x} \chi_{x x x}-6 y_{x x} \chi_{x x}-4 y_{x x x} \chi_{x},
$$

this leads to

$$
\begin{aligned}
\int_{N\left(\tau_{3}\right)} \theta^{2}\left|\tilde{y}_{t t}-\tilde{y}_{x x x x}\right|^{2} \mathrm{~d} x \mathrm{~d} t & =\int_{N\left(\tau_{3}\right)} \theta^{2}\left|y\left(\chi_{t t}-\chi_{x x x x}\right)+2 y_{t} \chi_{t}-4 y_{x} \chi_{x x x}-6 y_{x x} \chi_{x x}-4 y_{x x x} \chi_{x}\right|^{2} \mathrm{~d} x \mathrm{~d} t \\
& =\int_{N\left(\tau_{3}\right) \backslash N\left(\tau_{2}\right)} \theta^{2}\left|y\left(\chi_{t t}-\chi_{x x x x}\right)+2 y_{t} \chi_{t}-4 y_{x} \chi_{x x x}-6 y_{x x} \chi_{x x}-4 y_{x x x} \chi_{x}\right|^{2} \mathrm{~d} x \mathrm{~d} t \\
& \leq C \mathrm{e}^{2 \lambda \mathrm{e}^{h\left(a+\tau_{2}\right) \mu}} \int_{N\left(\tau_{3}\right) \backslash N\left(\tau_{2}\right)}\left(y^{2}+y_{t}^{2}+y_{x}^{2}+y_{x x}^{2}+y_{x x x}^{2}\right) \mathrm{d} x \mathrm{~d} t \\
& \leq C \mathrm{e}^{2 \lambda \mathrm{e}^{h\left(a+\tau_{2}\right) \mu}} \int_{N\left(\tau_{3}\right)}\left(y^{2}+y_{t}^{2}+y_{x}^{2}+y_{x x}^{2}+y_{x x x}^{2}\right) \mathrm{d} x \mathrm{~d} t \\
& =C \mathrm{e}^{2 \lambda \mathrm{e}^{h\left(a+\tau_{2}\right) \mu}}\|y\|_{\star, N\left(\tau_{3}\right)}^{2} .
\end{aligned}
$$

From the above estimates, we conclude that

$$
\lambda \mu^{2} \mathrm{e}^{h\left(a+\tau_{1}\right) \mu} \mathrm{e}^{2 \lambda \mathrm{e}^{h\left(a+\tau_{1}\right) \mu}}\|y\|_{\star, N\left(\tau_{1}\right)}^{2} \leq C\left(\lambda^{3} \mu^{5} \mathrm{e}^{3 \mu} \mathrm{e}^{2 \lambda \mathrm{e}^{\mu}}\left(\left\|y_{t}(0)\right\|_{L^{2}(\omega)}^{2}+\|y(0)\|_{H^{3}(\omega)}^{2}\right)+\mathrm{e}^{2 \lambda \mathrm{e}^{h\left(a+\tau_{2}\right) \mu}}\|y\|_{\star, N\left(\tau_{3}\right)}^{2}\right),
$$

namely,

$$
\begin{aligned}
\|y\|_{\star, N\left(\tau_{1}\right) \leq}^{2} \leq & C\left(\lambda^{2} \mu^{3} \mathrm{e}^{3 \mu-h\left(a+\tau_{1}\right) \mu} \mathrm{e}^{2 \lambda\left(\mathrm{e}^{\mu}-\mathrm{e}^{h\left(a+\tau_{1}\right) \mu}\right)}\left(\left\|y_{t}(0)\right\|_{L^{2}(\omega)}^{2}+\|y(0)\|_{H^{3}(\omega)}^{2}\right)\right. \\
& \left.+\lambda^{-1} \mu^{-2} \mathrm{e}^{-h\left(a+\tau_{1}\right) \mu} \mathrm{e}^{2 \lambda\left(\mathrm{e}^{h\left(a+\tau_{2}\right) \mu}-\mathrm{e}^{h\left(a+\tau_{1}\right) \mu}\right)}\|y\|_{\star, N\left(\tau_{3}\right)}^{2}\right) .
\end{aligned}
$$

Set

$$
\left\{\begin{array}{l}
\varepsilon=\lambda^{-1} \mu^{-2} \mathrm{e}^{-h\left(a+\tau_{1}\right) \mu} \mathrm{e}^{2 \lambda\left(\mathrm{e}^{h\left(a+\tau_{2}\right) \mu}-\mathrm{e}^{h\left(a+\tau_{1}\right) \mu}\right)} \\
\varepsilon_{0}=\lambda_{0}^{-1} \mu^{-2} \mathrm{e}^{-h\left(a+\tau_{1}\right) \mu} \mathrm{e}^{2 \lambda_{0}\left(\mathrm{e}^{h\left(a+\tau_{2}\right) \mu}-\mathrm{e}^{h\left(a+\tau_{1}\right) \mu}\right)} \\
k=\frac{\mathrm{e}^{\mu}-\mathrm{e}^{h\left(a+\tau_{1}\right) \mu}}{\mathrm{e}^{h\left(a+\tau_{1}\right) \mu}-\mathrm{e}^{h\left(a+\tau_{2}\right) \mu}}
\end{array}\right.
$$


It is clear that $1>h\left(a+\tau_{1}\right)>h\left(a+\tau_{2}\right)$, therefore, we can choose $\mu$ large enough such that $k>2$. Then for any $\varepsilon \in\left(0, \varepsilon_{0}\right]$ the following inequality holds:

$$
\|y\|_{\star, N\left(\tau_{1}\right)} \leq C \varepsilon\|y\|_{\star, Q_{0}}+C \varepsilon^{-k}\left(\left\|y_{t}(0)\right\|_{L^{2}(\omega)}+\|y(0)\|_{H^{3}(\omega)}\right) .
$$

Therefore, (3.14) holds for all $\varepsilon>0$. Further, if we let

$$
\beta_{1}=\frac{1}{1+k}, \varepsilon=\left(\frac{\left\|y_{t}(0)\right\|_{L^{2}(\omega)}+\|y(0)\|_{H^{3}(\omega)}}{\|y\|_{\star, Q_{0}}}\right)^{\beta_{1}},
$$

(3.13) follows immediately from (3.14).

There must be some open ball $B \subset N\left(\tau_{1}\right)$, it is easy to see that

$$
\|y\|_{\star, B} \leq C\left(\left\|y_{t}(0)\right\|_{L^{2}(\omega)}+\|y(0)\|_{H^{3}(\omega)}\right)^{\beta_{1}}\|y\|_{\star, Q_{0}}^{1-\beta_{1}} .
$$

Step 2. We shall prove that there exists a constant $\beta_{2} \in(0,1)$ such that

$$
\|y\|_{\star, \omega \times\left(\gamma, T_{0}-\gamma\right)} \leq C\|y\|_{\star, B}^{\beta_{2}}\|y\|_{\star, Q_{0}}^{1-\beta_{2}} .
$$

Indeed, let $B_{i}, i=1,2,3$ be three open balls with the properties $B_{1} \subset \subset B_{2} \subset \subset B_{3} \subset \subset Q_{0}$. Take $\eta \in C_{0}^{\infty}\left(Q_{0}\right)$ be valued in $(0,1)$ and $\eta \equiv 1$ in $B_{3}$.

Set

$$
\begin{aligned}
\psi_{3}(x, t) & =\left(x-x_{0}\right)^{2}-\widetilde{C}_{2}\left(t-\frac{T_{0}}{2}\right)^{2}+\widetilde{C}_{3}, \\
r_{1} & =\sup _{(x, t) \in B_{1}}\left(\left|x-x_{0}\right|^{2}+\left|t-t_{0}\right|^{2}\right)^{\frac{1}{2}}, \\
r_{2} & =\sup _{(x, t) \in B_{2}}\left|t-t_{0}\right|, \\
r_{3} & =\inf _{(x, t) \in Q_{0} \backslash B_{3}}\left|t-t_{0}\right|, \\
r_{Q_{0}} & =\sup _{(x, t) \in Q_{0} \backslash B_{3}}\left|x-x_{0}\right|,
\end{aligned}
$$

where $\left(x_{0}, t_{0}\right)$ is the center of $B_{1}$ and $\widetilde{C}_{2}, \widetilde{C}_{3}$ are large enough such that $\widetilde{C}_{2}\left(r_{3}^{2}-r_{2}^{2}\right) \geq r_{Q_{0}}^{2}$ and $\psi_{3} \geq 0$.

We here let $\psi(x, t)=\psi_{3}(x, t)$ in Proposition 2.1 and apply (2.1) to $\hat{y}=\eta y$ and $z=\theta \hat{y}$, then it follows that

$$
\begin{gathered}
\int_{Q_{0}}\left(\lambda^{3} \mu^{4} \varphi^{3} \psi_{3 x}^{4} z_{t}^{2}+\lambda^{7} \mu^{8} \varphi^{7} \psi_{3 x}^{8} z^{2}+\lambda^{5} \mu^{6} \varphi^{5} \psi_{3 x}^{8} z_{x}^{2}+\lambda^{3} \mu^{4} \varphi^{3} \psi_{3 x}^{4} z_{x x}^{2}+\lambda \mu^{2} \varphi \psi_{3 x}^{2} z_{x x x}^{2}+\lambda \mu^{2} \varphi \psi_{3 x}^{2} z_{x t}^{2}\right) \mathrm{d} x \mathrm{~d} t \\
\leq C\left(\int_{Q_{0}} \theta^{2}\left|\hat{y}_{t t}-\hat{y}_{x x x x}\right|^{2} \mathrm{~d} x \mathrm{~d} t+\int_{Q_{0}}\left(\lambda^{3} \mu^{3} \varphi^{3} z_{t}^{2}+\lambda^{7} \mu^{7} \varphi^{7} z^{2}\right.\right. \\
\left.\left.+\lambda^{5} \mu^{5} \varphi^{5} z_{x}^{2}+\lambda^{3} \mu^{3} \varphi^{3} z_{x x}^{2}+\lambda \mu \varphi z_{x x x}^{2}+\lambda \mu \varphi z_{x t}^{2}\right) \mathrm{~d} x \mathrm{~d} t\right) .
\end{gathered}
$$

By the same argument as in the proof of Lemma 3.2, there exists $\mu_{1}>0$ such that for $\mu \geq \mu_{1}$, we have

$$
\begin{array}{r}
\int_{Q_{0}}\left(\lambda^{3} \mu^{3} \varphi^{3} z_{t}^{2}+\lambda^{7} \mu^{7} \varphi^{7} z^{2}+\lambda^{5} \mu^{5} \varphi^{5} z_{x}^{2}+\lambda^{3} \mu^{3} \varphi^{3} z_{x x}^{2}+\lambda \mu \varphi z_{x x x}^{2}+\lambda \mu \varphi z_{x t}^{2}\right) \mathrm{d} x \mathrm{~d} t \\
\leq C\left(\int_{Q_{0}} \theta^{2}\left|\hat{y}_{t t}-\hat{y}_{x x x x}\right|^{2} \mathrm{~d} x \mathrm{~d} t+\int_{B_{1}}\left(\lambda^{3} \mu^{3} \varphi^{3} z_{t}^{2}+\lambda^{7} \mu^{7} \varphi^{7} z^{2}\right.\right. \\
\left.\left.+\lambda^{5} \mu^{5} \varphi^{5} z_{x}^{2}+\lambda^{3} \mu^{3} \varphi^{3} z_{x x}^{2}+\lambda \mu \varphi z_{x x x}^{2}+\lambda \mu \varphi z_{x t}^{2}\right) \mathrm{~d} x \mathrm{~d} t\right) .
\end{array}
$$


Taking into account the construction of $\psi_{3}$, we have

$$
\begin{aligned}
\int_{Q_{0}} & \left(\lambda^{3} \mu^{3} \varphi^{3} z_{t}^{2}+\lambda^{7} \mu^{7} \varphi^{7} z^{2}+\lambda^{5} \mu^{5} \varphi^{5} z_{x}^{2}+\lambda^{3} \mu^{3} \varphi^{3} z_{x x}^{2}+\lambda \mu \varphi z_{x x x}^{2}+\lambda \mu \varphi z_{x t}^{2}\right) \mathrm{d} x \mathrm{~d} t \\
& \geq C \int_{Q_{0}}\left(\lambda^{3} \mu^{3} \varphi^{3} \theta^{2} \hat{y}_{t}^{2}+\lambda^{7} \mu^{7} \varphi^{7} \theta^{2} \hat{y}^{2}+\lambda^{5} \mu^{5} \varphi^{5} \theta^{2} \hat{y}_{x}^{2}+\lambda^{3} \mu^{3} \varphi^{3} \theta^{2} \hat{y}_{x x}^{2}+\lambda \mu \varphi \theta^{2} \hat{y}_{x x x}^{2}+\lambda \mu \varphi \theta^{2} \hat{y}_{x t}^{2}\right) \mathrm{d} x \mathrm{~d} t \\
& \geq C \int_{B_{2}}\left(\lambda^{3} \mu^{3} \varphi^{3} \theta^{2} y_{t}^{2}+\lambda^{7} \mu^{7} \varphi^{7} \theta^{2} y^{2}+\lambda^{5} \mu^{5} \varphi^{5} \theta^{2} y_{x}^{2}+\lambda^{3} \mu^{3} \varphi^{3} \theta^{2} y_{x x}^{2}+\lambda \mu \varphi \theta^{2} y_{x x x}^{2}+\lambda \mu \varphi \theta^{2} y_{x t}^{2}\right) \mathrm{d} x \mathrm{~d} t \\
& \geq C \lambda \mu \mathrm{e}^{\mu\left(\widetilde{C}_{3}-\widetilde{C}_{2} r_{2}^{2}\right)} \mathrm{e}^{2 \lambda \mathrm{e}^{\mu\left(\widetilde{C}_{3}-\widetilde{C}_{2} r_{2}^{2}\right)}} \int_{B_{2}}\left(y_{t}^{2}+y^{2}+y_{x}^{2}+y_{x x}^{2}+y_{x x x}^{2}+y_{x t}^{2}\right) \mathrm{d} x \mathrm{~d} t \\
& =C \lambda \mu \mathrm{e}^{\mu\left(\widetilde{C}_{3}-\widetilde{C}_{2} r_{2}^{2}\right)} \mathrm{e}^{2 \lambda \mathrm{e}^{\mu\left(\tilde{C}_{3}-\tilde{C}_{2} r_{2}^{2}\right)}}\|y\|_{\star, B_{2}}^{2}
\end{aligned}
$$

and

$$
\begin{aligned}
\int_{B_{1}} & \left(\lambda^{3} \mu^{3} \varphi^{3} z_{t}^{2}+\lambda^{7} \mu^{7} \varphi^{7} z^{2}+\lambda^{5} \mu^{5} \varphi^{5} z_{x}^{2}+\lambda^{3} \mu^{3} \varphi^{3} z_{x x}^{2}+\lambda \mu \varphi z_{x x x}^{2}+\lambda \mu \varphi z_{x t}^{2}\right) \mathrm{d} x \mathrm{~d} t \\
& \leq C \int_{B_{1}}\left(\lambda^{3} \mu^{3} \varphi^{3} \theta^{2} y_{t}^{2}+\lambda^{7} \mu^{7} \varphi^{7} \theta^{2} y^{2}+\lambda^{5} \mu^{5} \varphi^{5} \theta^{2} y_{x}^{2}+\lambda^{3} \mu^{3} \varphi^{3} \theta^{2} y_{x x}^{2}+\lambda \mu \varphi \theta^{2} y_{x x x}^{2}+\lambda \mu \varphi \theta^{2} y_{x t}^{2}\right) \mathrm{d} x \mathrm{~d} t \\
& \leq C \lambda^{7} \mu^{7} \mathrm{e}^{7 \mu\left(r_{1}^{2}+\widetilde{C}_{3}\right)} \mathrm{e}^{2 \lambda \mathrm{e}^{\mu\left(r_{1}^{2}+\tilde{C}_{3}\right)}} \int_{B_{1}}\left(y_{t}^{2}+y^{2}+y_{x}^{2}+y_{x x}^{2}+y_{x x x}^{2}+y_{x t}^{2}\right) \mathrm{d} x \mathrm{~d} t \\
& =C \lambda^{7} \mu^{7} \mathrm{e}^{7 \mu\left(r_{1}^{2}+\widetilde{C}_{3}\right)} \mathrm{e}^{2 \lambda \mathrm{e}^{\mu\left(r_{1}^{2}+\tilde{C}_{3}\right)}\|y\|_{\star, B_{1}}^{2} .}
\end{aligned}
$$

Noting that $\hat{y}$ solves

$$
\hat{y}_{t t}-\hat{y}_{x x x x}=y\left(\eta_{t t}-\eta_{x x x x}\right)+y_{t} \cdot 2 \eta_{t}-y_{x} \cdot 4 \eta_{x x x}-y_{x x} \cdot 6 \eta_{x x}-y_{x x x} \cdot 4 \eta_{x},
$$

we have

$$
\begin{aligned}
\int_{Q_{0}} \theta^{2}\left|\hat{y}_{t t}-\hat{y}_{x x x x}\right|^{2} \mathrm{~d} x \mathrm{~d} t & \leq \int_{Q_{0}} \theta^{2}\left|y\left(\eta_{t t}-\eta_{x x x x}\right)+y_{t} \cdot 2 \eta_{t}-y_{x} \cdot 4 \eta_{x x x}-y_{x x} \cdot 6 \eta_{x x}-y_{x x x} \cdot 4 \eta_{x}\right|^{2} \mathrm{~d} x \mathrm{~d} t \\
& =\int_{Q_{0} \backslash B_{3}} \theta^{2}\left|y\left(\eta_{t t}-\eta_{x x x x}\right)+y_{t} \cdot 2 \eta_{t}-y_{x} \cdot 4 \eta_{x x x}-y_{x x} \cdot 6 \eta_{x x}-y_{x x x} \cdot 4 \eta_{x}\right|^{2} \mathrm{~d} x \mathrm{~d} t \\
& \leq C \mathrm{e}^{2 \lambda \mathrm{e}^{\mu\left(r_{Q_{0}}^{2}-\tilde{C}_{2} r_{3}^{2}+\tilde{C}_{3}\right)}}\|y\|_{\star, Q_{0}}^{2} .
\end{aligned}
$$

From the above estimates, we know that

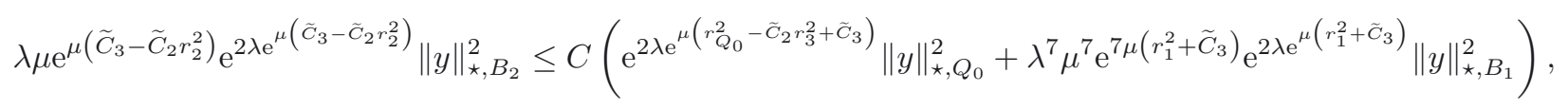

namely,

$$
\begin{aligned}
\|y\|_{\star, B_{2}}^{2} \leq & C\left(\lambda^{-1} \mu^{-1} \mathrm{e}^{-\left(\widetilde{C}_{3}-\widetilde{C}_{2} r_{2}^{2}\right) \mu} \mathrm{e}^{2 \lambda\left(\mathrm{e}^{\mu\left(r_{Q_{0}}^{2}-\widetilde{C}_{2} r_{3}^{2}+\widetilde{C}_{3}\right)}-\mathrm{e}^{\mu\left(\widetilde{C}_{3}-\widetilde{C}_{2} r_{2}^{2}\right)}\right)}\|y\|_{\star, Q_{0}}^{2}\right. \\
& \left.+\lambda^{6} \mu^{6} \mathrm{e}^{7 \mu\left(r_{1}^{2}+\widetilde{C}_{3}\right)-\mu\left(\widetilde{C}_{3}-\widetilde{C}_{2} r_{2}^{2}\right)} \mathrm{e}^{2 \lambda\left(\mathrm{e}^{\mu\left(r_{1}^{2}+\widetilde{C}_{3}\right)}-\mathrm{e}^{\mu\left(\widetilde{C}_{3}-\widetilde{C}_{2} r_{2}^{2}\right)}\right)}\|y\|_{\star, B_{1}}^{2}\right) .
\end{aligned}
$$


Set

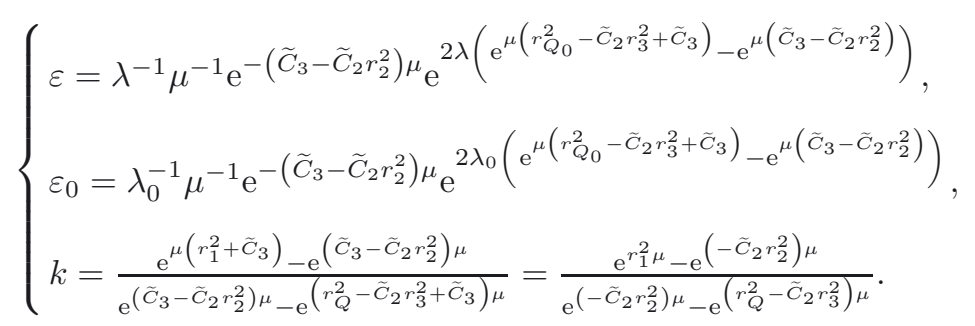

Note $\widetilde{C}_{2}\left(r_{3}^{2}-r_{2}^{2}\right)>r_{Q}^{2}$, therefore, we can choose $\mu$ large enough such that $k>6$.

By the same method as in Step 1, it is easy to see that

$$
\|y\|_{\star, B_{2}} \leq C\|y\|_{\star, B_{1}}^{\delta}\|y\|_{\star, Q_{0}}^{1-\delta},
$$

where $\delta \in(0,1)$.

For any open ball $B^{\prime} \subset \subset Q_{0}$, there is finite natural number $m$ and two sequences of open balls $\left\{B^{i}\right\}_{i=1}^{n}$ and $\left\{\widetilde{B}^{i}\right\}_{i=1}^{m}$ such that

$$
\left\{\begin{array}{l}
B^{\prime} \subset B^{1}, \widetilde{B}^{i} \subset \subset B^{i} \cap B^{i+1}, i=1, \ldots, m-1, \\
\widetilde{B}^{m} \subset \subset B^{m}, \widetilde{B}^{m} \subset B .
\end{array}\right.
$$

In view of (3.15), there must be a sequence $\left\{\delta_{i}\right\}_{i=1}^{m}$ such that

$$
\begin{aligned}
\|y\|_{\star, B^{\prime}} & \leq\|y\|_{\star, B^{1}} \leq C\|y\|_{\star, \widetilde{B}^{1}}^{\delta_{1}}\|y\|_{\star, Q_{0}}^{1-\delta_{1}} \leq C\|y\|_{\star, B^{2}}^{\delta_{1}}\|y\|_{\star, Q_{0}}^{1-\delta_{1}} \\
& \leq C\|y\|_{\star, \widetilde{B}^{2}}^{\delta_{1} \delta_{2}}\|y\|_{\star, Q_{0}}^{1-\delta_{1} \delta_{2}} \leq \ldots \leq C\|y\|_{\star, \widetilde{B}^{m}}^{\delta_{1} \delta_{2} \ldots \delta_{m}}\|y\|_{\star, Q_{0}}^{1-\delta_{1} \delta_{2} \ldots \delta_{m}} .
\end{aligned}
$$

Setting $\delta^{\prime}=\delta_{1} \delta_{2} \ldots \delta_{m}$, it follows that

$$
\|y\|_{\star, B^{\prime}} \leq C\|y\|_{\star, B}^{\delta^{\prime}}\|y\|_{\star, Q_{0}}^{1-\delta^{\prime}}
$$

Since $\omega \times\left(\gamma, T_{0}-\gamma\right) \subset \subset Q_{0}$, there must be a finite subcover of open balls, then from (3.16) we know there is a constant $0<\beta_{2}<1$ such that

$$
\|y\|_{\star, \omega \times\left(\gamma, T_{0}-\gamma\right)} \leq C\|y\|_{\star, B}^{\beta_{2}}\|y\|_{\star, Q_{0}}^{1-\beta_{2}} .
$$

Define $\alpha_{2}=\beta_{1} \beta_{2}$, we can deduce that

$$
\|y\|_{\star, \omega \times\left(\gamma, T_{0}-\gamma\right)} \leq C\left(\left\|y_{t}(0)\right\|_{L^{2}(\omega)}+\|y(0)\|_{H^{3}(\omega)}\right)^{\alpha_{2}}\|y\|_{\star, Q_{0}}^{1-\alpha_{2}} .
$$

Now we can prove Theorem 1.1.

Proof of Theorem 1.1. Following Lemma 3.2 and 3.3, there exists two constants $\alpha_{1}, \alpha_{2} \in(0,1)$ such that for any $y \in \mathcal{G}$ which solves (3.1), satisfy

$$
\|y\|_{L^{2}\left(I \times\left(T^{\prime}, T^{\prime \prime}\right)\right)} \leq C\|y\|_{\star, \omega \times\left(\gamma, T_{0}-\gamma\right)}^{\alpha_{1}}\|y\|_{\star, Q_{0}}^{1-\alpha_{1}}
$$

and

$$
\|y\|_{\star, \omega \times\left(\gamma, T_{0}-\gamma\right)} \leq C\left(\left\|y_{t}(0)\right\|_{L^{2}(\omega)}+\|y(0)\|_{H^{3}(\omega)}\right)^{\alpha_{2}}\|y\|_{\star, Q_{0}}^{1-\alpha_{2}}
$$

which conclude that

$$
\|y\|_{L^{2}\left(I \times\left(T^{\prime}, T^{\prime \prime}\right)\right)} \leq C\left(\left\|y_{t}(0)\right\|_{L^{2}(\omega)}+\|y(0)\|_{H^{3}(\omega)}\right)^{\alpha_{1} \alpha_{2}}\|y\|_{\star, Q_{0}}^{1-\alpha_{1} \alpha_{2}}
$$


Set $y(x, t)=\sum_{\lambda_{i}<r} \frac{s h t b_{i}}{b_{i}} a_{i} e_{i}$, where $b_{i}=\sqrt{\lambda_{i}}$ and $\frac{s h t b}{b}=t$ for $b=0$. Direct computation shows that $y$ given as above solves (3.1), which vanishes when $(x, t) \in \omega \times\{0\}$. It is obvious that both Rey and Imy satisfy (3.1). Applying (3.17) to Rey gives that

$$
\|\operatorname{Re} y\|_{L^{2}\left(I \times\left(T^{\prime}, T^{\prime \prime}\right)\right)} \leq C\left(\left\|\operatorname{Re} y_{t}(0)\right\|_{L^{2}(\omega)}+\|\operatorname{Re} y(0)\|_{H^{3}(\omega)}\right)^{\alpha_{1} \alpha_{2}}\|\operatorname{Re} y\|_{\star, Q_{0}}^{1-\alpha_{1} \alpha_{2}} .
$$

Since

$$
\begin{aligned}
\|\operatorname{Re} y\|_{\star, Q_{0}}^{2} & \leq C \mathrm{e}^{C \sqrt{r}} \sum_{\lambda_{i} \leq r}\left|\operatorname{Re} a_{i}\right|^{2}, \\
(\operatorname{Re} y)_{t}(x, 0) & =\sum_{\lambda_{i} \leq r} \operatorname{Re} a_{i} e_{i}, \\
(\operatorname{Re} y)(x, 0) & =(\operatorname{Re} y)_{x}(x, 0)=(\operatorname{Re} y)_{x x}(x, 0)=(\operatorname{Re} y)_{x x x}(x, 0)=0
\end{aligned}
$$

and

$$
\begin{aligned}
\|\operatorname{Re} y\|_{L^{2}\left(I \times\left(T^{\prime}, T^{\prime \prime}\right)\right)}^{2} & =\int_{T^{\prime}}^{T^{\prime \prime}} \int_{I}\left|\sum_{\lambda_{i} \leq r} \frac{s h t b_{i}}{b_{i}} \operatorname{Re} a_{i} e_{i}\right|^{2} \mathrm{~d} x \mathrm{~d} t \\
& =\sum_{\lambda_{i} \leq r}\left|\operatorname{Re} a_{i}\right|^{2} \int_{T^{\prime}}^{T^{\prime \prime}}\left|\frac{s h t b_{i}}{b_{i}}\right|^{2} \mathrm{~d} t \\
& \geq \sum_{\lambda_{i} \leq r}\left|\operatorname{Re} a_{i}\right|^{2} \int_{T^{\prime}}^{T^{\prime \prime}} t^{2} \mathrm{~d} t \\
& =C\left(T^{\prime}, T^{\prime \prime}\right) \sum_{\lambda_{i} \leq r}\left|\operatorname{Re} a_{i}\right|^{2}
\end{aligned}
$$

it is shown that

$$
\sum_{\lambda_{i} \leq r}\left|\operatorname{Re} a_{i}\right|^{2} \leq C \mathrm{e}^{C \sqrt{r}} \int_{\omega}\left|\sum_{\lambda_{i} \leq r} \operatorname{Re} a_{i} e_{i}\right|^{2} \mathrm{~d} x .
$$

By the same manner, we have

$$
\sum_{\lambda_{i} \leq r}\left|\operatorname{Im} a_{i}\right|^{2} \leq C \mathrm{e}^{C \sqrt{r}} \int_{\omega}\left|\sum_{\lambda_{i} \leq r} \operatorname{Im} a_{i} e_{i}\right|^{2} \mathrm{~d} x .
$$

This complete the proof with

$$
\sum_{\lambda_{i} \leq r}\left|a_{i}\right|^{2} \leq C_{1} \mathrm{e}^{C_{2} \sqrt{r}} \int_{\omega}\left|\sum_{\lambda_{i} \leq r} a_{i} e_{i}\right|^{2} \mathrm{~d} x .
$$

\section{Proof of Theorem 1.2}

The proof of Theorem 1.2 combines the ideas in $[8,9]$. First we give several lemmas. 
Lemma 4.1. ([6], pp. 256-257) For almost all $\tilde{t} \in E$, there exists a sequence of numbers $\left\{t_{i}\right\}_{i=1}^{\infty} \subset(0, T)$ such that

$$
\begin{array}{r}
t_{1}<t_{2}<\ldots t_{i}<t_{i+1}<\ldots<\tilde{t}, \quad t_{i} \rightarrow \tilde{t} \text { as } i \rightarrow \infty, \\
m\left(E \cap\left[t_{i}, t_{i+1}\right]\right) \geq \rho\left(t_{i+1}-t_{i}\right), \quad i=1,2, \ldots, \\
\frac{t_{i+1}-t_{i}}{t_{i+2}-t_{i+1}} \leq C_{0}, \quad i=1,2, \ldots,
\end{array}
$$

where $\rho$ and $C_{0}$ are two positive constants which are independent of $i$.

\section{Lemma 4.2.}

(a) For each $r \geq \lambda_{1}$, there exists a control $f_{r} \in L_{\mathcal{F}}^{\infty}\left(0, T ; L^{2}\left(\Omega ; X_{r}\right)\right)$ such that the solution $y$ of system (1.1) with $f=f_{r}$ satisfies $P_{r}(y(\cdot, T))=0$ in I, P-a.s. Moreover, $f_{r}$ verifies: If $2 \lambda_{1}^{\alpha}>\xi$, then

$$
\left\|f_{r}\right\|_{L_{\mathcal{F}}^{\infty}\left(0, T ; L^{2}\left(\Omega ; X_{r}\right)\right)}^{2} \leq \frac{C_{1} \mathrm{e}^{C_{2} \sqrt{r}}}{(m(E))^{2}} \mathbb{E}\left\|y_{0}\right\|_{L^{2}(I)}^{2} .
$$

For the general case, it holds that

$$
\left\|f_{r}\right\|_{L_{\mathcal{F}}^{\infty}\left(0, T ; L^{2}\left(\Omega ; X_{r}\right)\right)}^{2} \leq \frac{C_{1} \mathrm{e}^{C_{2} \sqrt{r}+\xi T}}{(m(E))^{2}} \mathbb{E}\left\|y_{0}\right\|_{L^{2}(I)}^{2} .
$$

(b) If $f \equiv 0$ in system (1.1), then for any $y_{0} \in L^{2}\left(\Omega, P, \mathcal{F}_{0} ; L^{2}(I)\right)$ with $P_{\lambda_{k-1}}\left(y_{0}\right)=0$ for some $k=2,3, \ldots$, the corresponding solution $y$ of system (1.1) satisfies

$$
\mathbb{E}\|y(t)\|_{L^{2}(I)}^{2} \leq \mathrm{e}^{-\left(2 \lambda_{k}^{\alpha}-\xi\right) t} \mathbb{E}\left\|y_{0}\right\|_{L^{2}(I)}^{2} .
$$

Proof.

(a) We first introduce the following backward stochastic fractional order Cahn-Hilliard equation

$$
\begin{cases}\mathrm{d} z-A^{\alpha} z=-a(t) Z \mathrm{~d} t+Z \mathrm{~d} B & \text { in } Q, \\ z(0, t)=0=z(1, t) & \text { in }(0, T), \\ z_{x}(0, t)=0=z_{x}(1, t) & \text { in }(0, T), \\ z(x, T)=z_{T}(x) & \text { in } I,\end{cases}
$$

where $z_{T} \in L^{2}\left(\Omega, P, \mathcal{F}_{T} ; X_{r}\right)$. According to $[2,12]$, (4.5) admits one and only one solution $(z, Z) \in$ $\left.L_{\mathcal{F}}^{2}\left(\Omega ; C\left([0, T] ; L^{2}(I)\right)\right) \times L_{\mathcal{F}}^{2}\left(0, T ; L^{2}(I)\right)\right)$.

Since $z_{T} \in L^{2}\left(\Omega, P, \mathcal{F}_{T} ; X_{r}\right), z_{T}$ can be written as $z_{T}=\sum_{\lambda_{i} \leq r} z_{T}^{i} e_{i}$ for a sequence of $\mathcal{F}_{T}$-measurable random variable $\left\{z_{T}^{i}\right\}_{\lambda_{i} \leq r}$. Then the solution $(z, Z)$ of (4.5) can be expressed as

$$
z=\sum_{\lambda_{i} \leq r} z_{i}(t) e_{i}, Z=\sum_{\lambda_{i} \leq r} Z_{i}(t) e_{i}
$$

where $z_{i}(\cdot) \in L_{\mathcal{F}}^{2}(\Omega ; C([0, T]))$ and $Z_{i}(\cdot) \in L_{\mathcal{F}}^{2}(0, T)\left(\lambda_{i} \leq r\right)$ satisfy the following equation

$$
\left\{\begin{array}{l}
\mathrm{d} z_{i}-\lambda_{i}^{\alpha} z_{i} \mathrm{~d} t=-a(t) Z_{i} \mathrm{~d} t+Z_{i} \mathrm{~d} B \\
z_{i}(T)=z_{T}^{i} .
\end{array} \text { in }(0, T),\right.
$$


It follows from Theorem 1.1 that

$$
\mathbb{E} \sum_{\lambda_{i} \leq r}\left|z_{i}(t)\right|^{2} \leq C_{1} \mathrm{e}^{C_{2} \sqrt{r}} \mathbb{E} \int_{\omega}\left|\sum_{\lambda_{i} \leq r} z_{i}(t) e_{i}\right|^{2} \mathrm{~d} x=C_{1} \mathrm{e}^{C_{2} \sqrt{r}} \mathbb{E} \int_{\omega}|z(t)|^{2} \mathrm{~d} x
$$

for any $t \in[0, T]$.

By Itô's formula, we see that $d|z|^{2}=2 z \mathrm{~d} z+(\mathrm{d} z)^{2}$. Hence we obtain that

$$
\begin{aligned}
\mathbb{E} \int_{I}|z(t)|^{2} \mathrm{~d} x-\mathbb{E} \int_{I}|z(0)|^{2} \mathrm{~d} x & =2 \mathbb{E} \int_{0}^{t} \sum_{\lambda_{i} \leq r} \lambda_{i}^{\alpha}\left|z_{i}(s)\right|^{2} \mathrm{~d} s+\mathbb{E} \int_{0}^{t} \int_{I}\left(-2 a(s) z Z+Z^{2}\right) \mathrm{d} x \mathrm{~d} s \\
& \geq 2 \mathbb{E} \int_{0}^{t} \sum_{\lambda_{i} \leq r} \lambda_{i}^{\alpha}\left|z_{i}(s)\right|^{2} \mathrm{~d} s-\mathbb{E} \int_{0}^{t} \int_{I}|a(s) z|^{2} \mathrm{~d} x \mathrm{~d} s \\
& \geq \mathbb{E} \int_{0}^{t} \sum_{\lambda_{i} \leq r}\left(2 \lambda_{i}^{\alpha}-\xi\right)\left|z_{i}(s)\right|^{2} \mathrm{~d} s \geq 0 .
\end{aligned}
$$

In view of (4.7)) and (4.8), we obtain that

$$
\mathbb{E} \int_{I} z^{2}(x, 0) \mathrm{d} x \leq C_{1} \mathrm{e}^{C_{2} \sqrt{r}} \mathbb{E} \int_{\omega} z^{2}(x, t) \mathrm{d} x
$$

for $t \in[0, T]$. Therefore,

$$
\int_{E}\left(\mathbb{E} \int_{I} z^{2}(x, 0) \mathrm{d} x\right)^{\frac{1}{2}} \mathrm{~d} t \leq\left(C_{1} \mathrm{e}^{C_{2} \sqrt{r}}\right)^{\frac{1}{2}} \int_{E}\left(\mathbb{E} \int_{\omega} z^{2}(x, t) \mathrm{d} x\right)^{\frac{1}{2}} \mathrm{~d} t .
$$

Hence we deduce that for each $z_{T} \in L^{2}\left(\Omega, P, \mathcal{F}_{T} ; X_{r}\right)$,

$$
\begin{aligned}
\mathbb{E} \int_{I} z^{2}(x, 0) \mathrm{d} x & \leq \frac{C_{1} \mathrm{e}^{C_{2} \sqrt{r}}}{(m(E))^{2}}\left(\int_{0}^{T}\left(\mathbb{E} \int_{I} \chi_{E} \chi_{\omega} z^{2}(x, t) \mathrm{d} x\right)^{\frac{1}{2}} \mathrm{~d} t\right)^{2} \\
& =\frac{C_{1} \mathrm{e}^{C_{2} \sqrt{r}}}{(m(E))^{2}}\left\|\chi_{E} \chi_{\omega} z\right\|_{L_{\mathcal{F}}^{1}\left(0, T ; L^{2}\left(\Omega ; L^{2}(I)\right)\right)}^{2} .
\end{aligned}
$$

According to dual argument and a Riesz-type Representation Theorem, one can get the first result in part (a) by similar argument as in [9].

Applying Itô's formula to $\mathrm{e}^{\tau t}|z|^{2}$, the second result in part a) can be obtained following the above methods with minor changes.

(b) Since $y_{0} \in L^{2}\left(\Omega, \mathcal{F}_{0}, P ; L^{2}(I)\right)$ with $P_{\lambda_{k-1}}\left(y_{0}\right)=0$, $y_{0}$ can be written as $y_{0}=\sum_{i=k}^{\infty} y_{0}^{i} e_{i}$ for suitable $y_{0}^{i} \in L^{2}\left(\Omega, \mathcal{F}_{0}, P\right)$. Thus, the solution $y$ of system (4.7) can be expressed as

$$
y=\sum_{i=k}^{\infty} y^{i}(t) e_{i}
$$

where $y^{i} \in L_{\mathcal{F}}^{2}(\Omega ; C([0, T]))$ solves the following equation

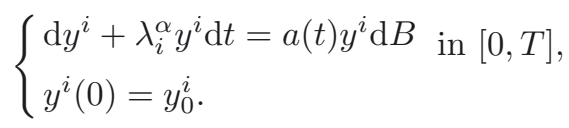


Applying Itô's formula to $\mathrm{e}^{\left(2 \lambda_{k}^{\alpha}-\xi\right) t}|y(t)|^{2}$, we have that

$$
\mathrm{d}\left(\mathrm{e}^{\left(2 \lambda_{k}^{\alpha}-\xi\right) t}|y(t)|^{2}\right)=\mathrm{e}^{\left(2 \lambda_{k}^{\alpha}-\xi\right) t} 2 y \mathrm{~d} y+\mathrm{e}^{\left(2 \lambda_{k}^{\alpha}-\xi\right) t}(\mathrm{~d} y)^{2}+\left(2 \lambda_{k}^{\alpha}-\xi\right) \mathrm{e}^{\left(2 \lambda_{k}^{\alpha}-\xi\right) t} y^{2} \mathrm{~d} t .
$$

Hence it is clear that

$$
\begin{aligned}
\mathbb{E} \int_{I} \mathrm{e}^{\left(2 \lambda_{k}^{\alpha}-\xi\right) t}|y(t)|^{2} \mathrm{~d} x-\mathbb{E} \int_{I}|y(0)|^{2} \mathrm{~d} x= & \mathbb{E} \int_{0}^{T} \mathrm{e}^{\left(2 \lambda_{k}^{\alpha}-\xi\right) s} \sum_{i=k}^{\infty}\left(-2 \lambda_{i}^{\alpha}\right)\left|y^{i}\right|^{2} \mathrm{~d} s \\
& +\mathbb{E} \int_{0}^{T} \int_{I} \mathrm{e}^{\left(2 \lambda_{k}^{\alpha}-\xi\right) s} a^{2}(s)|y|^{2} \mathrm{~d} x \mathrm{~d} s \\
& +\left(2 \lambda_{k}^{\alpha}-\xi\right) \mathbb{E} \int_{0}^{T} \int_{I} \mathrm{e}^{\left(2 \lambda_{k}^{\alpha}-\xi\right) s}|y|^{2} \mathrm{~d} x \mathrm{~d} s \leq 0
\end{aligned}
$$

which gives the desired estimate (4.4) immediately.

Proof of Theorem 1.2. By same idea in [9], without loss of generality, in what follows we assume that $\lambda_{1}^{\alpha} \geq \xi$ and $C_{1} \geq 1$.

By Lemma 4.1, we can take a number $\tilde{t} \in E$ with $\tilde{t}<T$ and a sequence $\left\{t_{N}\right\}_{N=1}^{\infty} \subseteq(0, T)$ such that (4.1)-(4.3) hold for some positive numbers $\rho$ and $C_{0}$, and such that $\tilde{t}-t_{1} \leq \min \left\{\lambda_{1}^{\alpha}, 1\right\}$.

First, we take $\tilde{y}_{0}(x)$ to be $q\left(x, t_{1}\right)$, where $q$ is the solution to the following equation

$$
\begin{cases}\mathrm{d} q+A^{\alpha} q \mathrm{~d} t=a(t) q \mathrm{~d} B & \text { in } I \times\left(0, t_{1}\right), \\ q(0, t)=0=q(1, t) & \text { in }\left(0, t_{1}\right), \\ q_{x}(0, t)=0=q_{x}(1, t) & \text { in }\left(0, t_{1}\right) \\ q(x, 0)=y_{0}(x) & \text { in } I .\end{cases}
$$

Next, we will show that there exists a control $\tilde{f} \in L_{\mathcal{F}}^{\infty}\left(t_{1}, \tilde{t} ; L^{2}\left(\Omega ; L^{2}(I)\right)\right)$ such that the solution $\tilde{y}$ of

$$
\begin{cases}\mathrm{d} \tilde{y}+A^{\alpha} \tilde{y} \mathrm{~d} t=a(t) \tilde{y} \mathrm{~d} B+\chi_{\omega} \chi_{E} \tilde{f} \mathrm{~d} t & \text { in } I \times\left(t_{1}, \tilde{t}\right), \\ \tilde{y}(0, t)=0=\tilde{y}(1, t) & \text { in }\left(t_{1}, \tilde{t}\right), \\ \tilde{y}_{x}(0, t)=0=\tilde{y}_{x}(1, t) & \text { in }\left(t_{1}, \tilde{t}\right), \\ \tilde{y}\left(x, t_{1}\right)=\tilde{y}_{0}(x) & \text { in } I,\end{cases}
$$

satisfying $\tilde{y}(\tilde{t})=0$ in $I, P$-a.s and

$$
\|\tilde{f}\|_{L_{\mathcal{F}}^{\infty}\left(t_{1}, \tilde{t} ; L^{2}\left(\Omega ; L^{2}(I)\right)\right)}^{2} \leq L \mathbb{E}\left\|\tilde{y}_{0}\right\|_{L^{2}(I)}^{2}
$$

where $L$ is a constant independent of $y_{0}$.

Indeed, set $K_{N}=\left[t_{2 N-1}, t_{2 N}\right]$ and $J_{N}=\left[t_{2 N}, t_{2 N+1}\right]$ for $N=1,2, \ldots$, then

$$
\left[t_{1}, \tilde{t}\right)=\bigcup_{N=1}^{\infty}\left(K_{N} \cup J_{N}\right) \text {. }
$$

It is clear that $m\left(E \cap K_{N}\right)>0$ and $m\left(E \cap J_{N}\right)>0$ for all $N \geq 1$.

According to Lemmas 4.1 and 4.2 and the same argument in proof of Theorem 1.1 in [9], we control equation (4.11) on $K_{N}$ corresponding a control $f_{N}$ such that $P_{\bar{r}_{N}}\left(y_{N}\left(\cdot, t_{2 N}\right)\right)=0$ and

$$
\left\|f_{N}\right\|_{L_{\mathcal{F}}^{\infty}\left(t_{2 N-1}, t_{2 N} ; L^{2}\left(\Omega ; L^{2}(I)\right)\right)}^{2} \leq 2^{N-1}\left(\frac{C_{1}}{\rho^{2}\left(t_{2}-t_{1}\right)^{2}}\right)^{N} C_{0}^{4} C_{0}^{4 \times 2} \ldots C_{0}^{4 \times(N-1)} \alpha_{1} \alpha_{2} \ldots \alpha_{N} \mathbb{E}\left\|\tilde{y}_{0}\right\|_{L^{2}(I)}^{2},
$$


where

$$
\begin{aligned}
& \alpha_{N}=\left\{\begin{array}{lr}
\mathrm{e}^{C_{2} \sqrt{\bar{r}_{1}}}, & N=1, \\
\mathrm{e}^{C_{2} \sqrt{\bar{r}_{N}}} \mathrm{e}^{\left(-2 \bar{r}_{N-1}^{\alpha}+\xi\right)\left(t_{3}-t_{2}\right) C_{0}^{-2(N-2)},} & N \geq 2,
\end{array}\right. \\
& \bar{r}_{N}=\left(\bar{C} \tilde{C}^{N-1}+\xi\right)^{\frac{2}{b}}, \quad N \geq 1, \\
& \bar{C}=\frac{2}{t_{3}-t_{2}}, \quad \tilde{C}=\frac{2 C_{1}}{\rho^{2}\left(t_{2}-t_{1}\right)^{2}} C_{0}^{2}, \quad b=\alpha-\frac{1}{2} .
\end{aligned}
$$

On the other hand, we let the equation evolve freely on $J_{N}$.

Now, we prove the existence of the constant $L$ in (4.12).

It is clear that

$$
\left\|f_{N}\right\|_{L_{\mathcal{F}}^{\infty}\left(t_{2 N-1}, t_{2 N} ; L^{2}\left(\Omega ; L^{2}(I)\right)\right)}^{2} \leq \tilde{C}^{N(N-1)} \alpha_{1} \alpha_{2} \ldots \alpha_{N} \mathbb{E}\left\|\tilde{y}_{0}\right\|_{L^{2}(I)}^{2},
$$

where $N>1$, and $2^{\frac{2}{b}} \leq \bar{r}_{1}<\bar{r}_{2}<\ldots<\bar{r}_{N} \rightarrow \infty$ as $N \rightarrow \infty$. Moreover,we have

$$
\left(\bar{r}_{N-1}\right)^{\frac{b}{2}}\left(t_{3}-t_{2}\right) C_{0}^{-2(N-2)}-\xi\left(t_{3}-t_{2}\right) C_{0}^{-2(N-2)} \geq 2, \quad N \geq 2,
$$

therefore

$$
\mathrm{e}^{-\left(2 \bar{r}_{N-1}^{\alpha}-\xi\right)\left(t_{3}-t_{2}\right) C_{0}^{-2(N-2)}} \leq \mathrm{e}^{-4 \bar{r}_{N-1}^{\frac{b+1}{2}}}, \quad N \geq 2 .
$$

Note that

$$
\tilde{C}^{N(N-1)} \mathrm{e}^{-\frac{\bar{r}_{N-1}}{2}}=\frac{\tilde{C}^{N(N-1)}}{\left(\mathrm{e}^{\frac{b}{\bar{r}_{N-1}^{2}}}\right)^{\bar{r}_{N-1}^{\frac{1}{2}}}} \leq \frac{\tilde{C}^{N(N-1)}}{\left(\mathrm{e}^{2 \tilde{C}^{N-2}}\right)^{\bar{r}_{N-1}^{\frac{1}{2}}}} \leq \frac{\tilde{C}^{N(N-1)}}{\left(\tilde{C}^{(N-2) 2 \bar{r}_{N-1}^{\frac{1}{2}}}\right.}
$$

and

$$
\mathrm{e}^{C_{2} \sqrt{\bar{r}_{N}}} \mathrm{e}^{-\bar{r}_{N-1}^{\frac{b+1}{2}}} \leq C(b) \mathrm{e}^{\tilde{C}^{\frac{N}{b}}-\tilde{C}^{\frac{N(b+1)}{b}}}
$$

for each $N \geq 2$, where $C(b)$ is a positive constant depend on $b$. We derive from the definition of $\bar{r}_{N}$ that there exists a natural number $N_{0}$, such that for all $N \geq N_{0}$,

$$
\tilde{C}^{N(N-1)} \mathrm{e}^{-\frac{b+1}{2}} \leq 1 \text { and } \mathrm{e}^{C_{2} \sqrt{\bar{r}_{N}}} \mathrm{e}^{-\frac{b+1}{2}} \leq 1 .
$$

Combining (4.13) and (4.14), we see that for all $N \geq N_{0}$,

$$
\begin{aligned}
\tilde{C}^{N(N-1)} \alpha_{N} & =\tilde{C}^{N(N-1)} \mathrm{e}^{C_{2} \sqrt{r_{N}}} \mathrm{e}^{\left(-2 \bar{r}_{N-1}^{\alpha}+\xi\right)\left(t_{3}-t_{2}\right) C_{0}^{-2(N-2)}} \\
& \leq \tilde{C}^{N(N-1)} \mathrm{e}^{C_{2} \sqrt{\bar{r}_{N}}} \mathrm{e}^{-4 \bar{r}_{N-1}^{\frac{b+1}{2}}} \\
& =\tilde{C}^{N(N-1)} \mathrm{e}^{-\bar{r}_{N-1}^{\frac{b+1}{2}}} \cdot \mathrm{e}^{C_{2} \sqrt{\bar{r}_{N}}} \mathrm{e}^{-\bar{r}_{N-1}^{\frac{b+1}{2}}} \cdot \mathrm{e}^{-2 \bar{r}_{N-1}^{\frac{b+1}{2}}} \\
& \leq 1 .
\end{aligned}
$$

Moreover, it is obviously that $\alpha_{N} \leq 1$ for any $N \geq N_{0}$. We set

$$
L=\max \left\{\tilde{C}^{N(N-1)} \alpha_{1} \alpha_{2} \ldots \alpha_{N}, 1 \leq N \leq N_{0}\right\}<\infty .
$$

Then we can obtain that

$$
\left\|f_{N}\right\|_{L_{\mathcal{F}}^{\infty}\left(t_{2 N-1}, t_{2 N} ; L^{2}\left(\Omega ; L^{2}(I)\right)\right)}^{2} \leq L \mathbb{E}\left\|\tilde{y}_{0}\right\|_{L^{2}(I)}^{2},
$$


where $N \geq 1$. We now contruct $\tilde{f}$ by setting

$$
\tilde{f}= \begin{cases}f_{N}(x, t) & x \in I, t \in K_{N}, N \geq 1 \\ 0 & x \in I, t \in J_{N}, N \geq 1 .\end{cases}
$$

It is clear that $\tilde{f} \in L_{\mathcal{F}}^{\infty}\left(t_{1}, \tilde{t} ; L^{2}\left(\Omega ; L^{2}(I)\right)\right)$ and satisfies the estimate

$$
\|\tilde{f}\|_{L_{\mathcal{F}}^{\infty}\left(t_{1}, \tilde{t} ; L^{2}\left(\Omega ; L^{2}(I)\right)\right)}^{2} \leq L \mathbb{E}\left\|\tilde{y}_{0}\right\|_{L^{2}(I)}^{2}
$$

Arguing as in $[9,10]$, we can obtain $\tilde{y}(x, \tilde{t})=0$ in $I, P$-a.s.

Finally, construct a control $f$ by setting

$$
f= \begin{cases}0 & \text { in } I \times\left(0, t_{1}\right), \\ \tilde{f}(x, t) & \text { in } I \times\left(t_{1}, \tilde{t}\right), \\ 0 & \text { in } I \times(\tilde{t}, T) .\end{cases}
$$

It is clear that $f \in L_{\mathcal{F}}^{\infty}\left(0, T ; L^{2}\left(\Omega ; L^{2}(I)\right)\right)$ and that the corresponding solution $y$ of $(1.1)$ satisfies $y(T)=0$ in $I$, $P$-a.s. Moreover, the control $f$ satisfies the estimate

$$
\|f\|_{L_{\mathcal{F}}^{\infty}\left(0, T ; L^{2}\left(\Omega ; L^{2}(I)\right)\right)}^{2} \leq L \mathbb{E}\left\|y_{0}\right\|_{L^{2}(I)}^{2}
$$

Acknowledgements. I would like to thank the referee and the editor for their careful comments and useful suggestions. I sincerely thank Professor Yong Li for many useful suggestions and help.

\section{REFERENCES}

[1] G. Da Prato and J. Zabczyk, Stochastic Equations in Infinite Dimensions. Cambridge University Press, Cambridge (1992).

[2] Y. Hu and S. Peng, Adapted solution of backward stochastic evolution equations. Stoch. Anal. Appl. 9 (1991) $445-459$.

[3] D. Jerison and G. Lebeau, Nodal sets of sums of eigenfunctions, in: Harmonic Analysis and Partial Differential Equations. Chicago Lectures in Math. Univ. Chicago Press, Chicago, IL (1999) 223-239.

[4] G. Lebeau and L. Robbiano, Contrôle exact de l'équation de la chaleur. Commun. Partial Differ. Equ. 20 (1995) 335-336.

[5] G. Lebeau and E. Zuazua, Null controllability of a system of linear thermoelasticity. Arch. Rational Mech. Anal. 141 (1998) $297-329$.

[6] J.L. Lions, Optimal Control of Systems Governed by Partial Differential Equations. Springer-Verlag, Berlin, Heidelberg, New York (1971).

[7] Q. Lü, Observation and control for stochastic partial differential equations. Ph.D. thesis, School of Mathematics. Sichuan University, Chengdu (2010).

[8] Q. Lü, Bang-bang principle of time optimal controls and null controllability of fractional order parabolic equations. Acta Math. Sin. (Engl. Ser.) 26 (2010) 2377-2386.

[9] Q. Lü, Some results on the controllability of forward stochastic heat equations with control on the drift. J. Funct. Anal. 260 (2011) 832-851.

[10] Q. Lü and G. Wang, On the existence of time optimal controls with constraints of the rectangular type for heat equations. SIAM J. Control Optim. 49 (2011) 1124-1149.

[11] Q. Lü and Z. Yin, The $L^{\infty}$-null controllability of parabolic equation with equivalued surface boundary conditions. Asymptot. Anal. 83 (2013) 355-378.

[12] N.I. Mahmudov and M.A. McKibben, On backward stochastic evolution equations in Hilbert spaces and optimal control. Nonlinear Anal. 67 (2007) 1260-1274.

[13] M. Renardy and R.C. Rogers, An Introduction to Partial Differential Equations, 2nd edn. Vol. 13 of Texts in Applied Mathematics. Springer-Verlag, New York (2004).

[14] G. Wang, $L^{\infty}$-null controllability for the heat equation and its consequences for the time optimal control problem. SIAM J. Control Optim. 47 (2008) 1701-1720.

[15] Z.C. Zhou, Observability estimate and null controllability for one-dimensional fourth order parabolic equation. Taiwanese J. Math. 16 (2012) 1991-2017. 